\title{
Dynamique de la processionnaire du pin dans la vallée de Niolo en Corse au cours des cycles 1965-1966, 1967-1968, 1969-1970. Rôle de certains caractères du milieu forestier
}

\author{
C. GERI \\ I.N.R.A., Station de Zoologie et de Biocénotique forestières \\ Centre de Recherches d'Orléans, Ardon, F 45160 Olivet
}

\section{Résumé}

Cet article fait le point des informations d'ordre démographique recueillies au cours de trois cycles d'étude 1965-1966, 1967-1968 et 1969-1970, sur la processionnaire du pin dans la vallée du Niolo en Corse.

L'utilisation simultanée de deux niveaux d'investigation et le recoupement de données acquises d'une part à l'échelle parcellaire, d'autre part au niveau du massif forestier permettent d'avoir sinon des preuves absolues, du moins des hypothèses largement étayées sur la façon dont s'est produite l'évolution de l'insecte au cours de ces six années.

Les résultats permettent en effet de suivre les fluctuations de population de la vallée avec une bonne précision statistique (coefficient de variation de 5 p. 100 en moyenne) et de mettre en évidence les faits suivants :

- le niveau de population correspondant à une culmination de gradation en 1966 (8 800000 nids) s'est progressivement abaissé en 1968 (6800000 nids) et en 1970 (2900 000 nids);

- les zones les plus peuplées durant l'annće 1966 furent celles où la réduction de population fut la plus importante au cours des années suivantes;

- les fortes populations de 1965-1966 furent accompagnées de la présence d'une virose à Smithiavirus pityocampa Vago. Celles-ci, dont la manifestation épidémique fut limitée aux zones de basse altitude ou bien exposées, ne fut retrouvée qu'à l'état endémique par la suite;

- les expositions nord-ouest sont les moins peuplées, et les expositions sud-est les plus favorables à la processionnaire ;

- durant les six années d'observation, il n'y eut jamais au cours des premiers stades de mortalité susceptible d'avoir une action significative sur l'évolution de la population;

- de même, les parasites de la phase aérienne de la processionnaire, en particulier les parasites des œufs ne paraissent pas avoir eu une action déterminante sur la mortalité enregistrée ;

- celle-ci au cours du cycle 1965-1966 fut essentiellement préhivernale, liée à la présence de virose dans un certain nombre de parcelles et d'autant plus importante que les parcelles étaient surpeuplées ;

- au cours du cycle 1969-1970, elle fut au contraire hivernale et indépendance du niveau de population. La réduction de population fut d'autant plus marquée sur les parcelles qui étaient exposées au Nord et à une altitude élevée; 
- le cycle 1968-1969 représente une situation intermédiaire. Les chenilles furent affectées par un hiver rigoureux mais dans un certain nombre de parcelles, on enregistra une mortalité préhivernale due à la surpopulation et du même type que celle de 1965 ;

- les analyses effectuées montrent, en outre, les liaisons étroites qui existent entre les niveaux de population de l'insecte et les principales caractéristiques du milieu forestier. Ces relations méritent d'être approfondies pour constituer des forêts qui, par elles-mêmes, se défendent mieux contre les attaques de processionnaires.

Les études sur la processionnaire du pin (Thaumetopoea pityocampa Schiff.) entreprises en 1965 dans la vallée du Niolo, en Corse, ont fait l'objet d'une thèse et d'une note plus synthétique en 1980.

Ce travail de dynamique des populations fait appel aux techniques d'études des populations dans leur milieu naturel qui ont été utilisées pour l'étude des insectes forestiers dans un certain nombre de pays où les forêts ont une importance économique appréciablc. (On se reportera notamment aux travaux de R.F. Morris \& C.A. Mill.er, 1954 ; R.F. Morris, 1957, 1959, 1969 ; R.F. Morris et al., 1963 ; R.W. Campbell, G.C. Varley \& C.R. Gradwell, 1960 ; C. Auer, 1968).

La méthodologie s'inspire essentiellement des techniques de tables de mortalité préconisées par R.F. MorRis et des recensements menés sur des vallées entières par C. Auer. Les résultats de ces deux approches très complémentaires et pourtant toujours utilisés isolément jusqu'à présent sont analysés comparativement. En outre, pour chaque génération, en sus des phénomènes démographiques proprement dits (mortalité, fécondité...), une attention particulière est apportée aux conditions physiques du milieu, à la structure et à la composition spécifique des peuplements forestiers pour rendre compte des fluctuations enregistrées.

Les objectifs de l'étude, la description du milieu étudié et les méthodes utilisées ont déjà été décrits dans cette revue (Dussausoy \& Geri, 1969) et aux Annales de Zoologie (GERI, 1971). Nous ne les rappellerons que très brièvement.

- Le but de notre travail est d'apprécier les fluctuations de populations de la processionnaire et d'analyser les facteurs qui les provoquent afin de prévoir les pullulations, de rationaliser les interventions, voire d'aménager le milieu pour qu'il se défende mieux par lui-même vis-à-vis des attaques de cet insecte.

- L'étude de la dynamique de la processionnaire est effectuée, d'une part, au moyen de dénombrements stade par stade des populations, et par des observations comparatives sur les principaux facteurs de régulation dans des parcelles de quelques hectares, d'autre part par des enquêtes sur l'ensemble des massifs forestiers permettant d'établir à chaque cycle, par dénombrements des nids d'hiver définitifs, des estimations représentatives de la vallée et de ses principaux peuplements.

- La population étudiée occupe tout l'ensemble forestier de la vallée du Niolo située au cœur de la chaîne centrale de la Corse ; cette vallée isolée par des massifs montagneux importants (Paglia Orba, $2525 \mathrm{~m}$; Monte Cinto, $2710 \mathrm{~m}$; Punta Artica, $2329 \mathrm{~m}$ ) comprend environ 6000 ha de pins laricios en peuplements presque purs qui s'étendent de $900 \mathrm{~m}$ jusqu'aux crêtes déboisées $(1400$ à $1600 \mathrm{~m}$ suivant les expositions). 
- En raison de l'intervention d'une diapause, le régime de la processionnaire du pin est, dans la vallée du Niolo, à peu près strictement biennal. Chaque «cycle " est séparé du suivant par une année sans pullulation et la ponte a lieu dans le Niolo au cours des années impaires $(1965,1967,1969)$.

Dans cet article, il nous a paru utile de reprendre les éléments de l'étude des trois premiers cycles biologiques de l'insecte (1965-1966, 1967-1968, 1969-1970). C'est, en effet, au cours de ces années-là, qu'ont eu lieu les investigations les plus détaillées sur l'évolution des populations de la processionnaire du pin dans cette vallée. Le bilan des deux approches méthodologiques que nous avons utilisées avait alors été effectué et certaines caractéristiques de la dynamique de l'insecte dans les zones d'altitude de la Corse avaient été mises en évidence. Ces conclusions n'ont pas été mises en défaut après plus de dix ans d'observations biologiques et de recensement des populations; elles ont, au contraire, largement contribué à la compréhension de l'évolution de la processionnaire non seulement dans la vallée du Niolo, mais sur l'ensemble de la Corse et dans d'autres régions.

La phase aérienne du développement de la processionnaire est seule prise en considération; mais les résultats sur la mortalité au cours de la vie souterraine de rinsecte et l'intensité des phénomènes de diapause (C. Geri, 1980) n’affectent pas de façon notable les conclusions formulées ici.

\section{1. Étude stade par stade des populations de chaque cycle biologique}

\subsection{Méthodes}

Des prélèvements sont effectués dans 5 parcelles de quelques hectares échelonnés en altitude dans le massif forestier :
PK 51
$950 \mathrm{~m}$ - Exposition E.S.E.
Mezza-Luna
$1030 \mathrm{~m}$ - Exposition N.
Pente des Genêts
$1150 \mathrm{~m}$ - Exposition S.E.
Castellacce-Bas
$1350 \mathrm{~m}$ - Exposition S.E.
Castellacce-Haut
$1370 \mathrm{~m}$ - Exposition E.N.E.

Toutes ces parcelles sont constituées par des peuplements clairs fortement infestés au cours des pullulations de chenilles sauf Mezza-Luna, ravin occupé par une régénération sous-futaie peu attaquée par les processionnaires.

Les techniques utilisées au cours du cycle 1969-1970 furent identiques à celles des années précédentes avec de petites modifications concernant le nombre et l'intensité des prélèvements, destinées à assurer une meilleure représentativité de l'échantillon.

La population initiale (œufs) est déterminée sur un échantillon de 15 à 45 pins, tirés au hasard dans chaque parcelle, à chaque cycle, et la population survivante à chaque stade est dénombrée sur un sous-échantillonnage de ces pins ( 3 à 9 pins).

Seules les données relatives à la population de la processionnaire ont fait l'objet d'une analyse statistique; les informations concernant la fécondité et le pourcentage de mortalité dû aux différents facteurs biotiques ne sont interprétées qu'en tant que 
valeurs moyennes, comparables d'un biotope à l'autre et d'une génération à l'autre, les divers prélèvements et observations étant toujours réalisés de façon identique et de façon à assurer la meilleure représentativité des phénomènes étudiés.

L'évolution de la population larvaire avait été étudiée pour les deux premiers cycles par l'analyse de covariance qui permet de tester les différences entre les prélèvements pour une même population initiale d'œufs. La validité de cette méthode suppose toutefois l'homogénéité de la régression entre le nombre de chenilles et le nombre d'œufs pour les différents prélèvements. Or, au fur et à mesure des prélèvements, la pente de la droite de régression diminue progressivement et la diminution du coefficient de corrélation traduit la perte de liaison entre l'importance numérique de la population de chenilles et celle de la population d'œufs initiale correspondante. Ce fait, confirmé au cours du troisième cycle étudié paraît se retrouver généralement chez les défoliateurs forestiers (voir notamment R.F. MoRRIs, 1955).

Il semble que ces modifications brutales de la pente de la droite de régression et du coefficient de corrélation reflètent précisément les périodes du cycle biologique où la mortalité affecte le plus la population.

L'analyse des données a donc été reprise selon une méthode proposée par J. ARNoux, Directeur du Service de Biométrie de l'I.N.R.A. à Versailles; cette méthode permet de classer les prélèvements en groupes tels que la régression soit la plus homogène possible à l'intérieur d'eux-mêmes, et présente le maximum de différences d'un groupe à l'autre. Cette méthode est dérivée du test d'homogénéité de régression déjà évoqué dans une note antérieure (C. GERI, 1971) et dont le principe peut être retrouvé dans SNEdecor (1962). Elle consiste à décomposer la somme des carrés qui traduit l'écart des régressions des différents prélèvements avec la régression commune, en deux termes relatifs, l'un à la différence entre les groupes ainsi composés, l'autre à ce qui découle de l'hétérogénéité de la régression à l'intérieur de ces groupes.

L'analyse est complétée par le calcul de l'estimation $\bar{y}^{\prime}$ du nombre moyen de chenilles à chaque prélèvement sur un pin possédant une population d'œufs égale à la moyenne de celles observées sur tous les pins échantillonnés, selon la formule :

$$
\left(\overline{\mathrm{y}}--\overline{\mathrm{y}}^{\prime}\right)=\mathrm{b}(\overline{\mathrm{x}}-\mathrm{X}) \text {, }
$$

utilisée notamment par M.H. Hansen, W.N. Hurwitz, W.G. Madow (1953), où :

$\bar{y}$ représente le nombre moyen de chenilles par pin dans le sous-échantillon où les chenilles ont été dénombrées,

x le nombre moyen d'œufs correspondant à $\bar{y}$,

$X$ le nombre moyen d'œufs sur tous les arbres échantillonnés

et $\mathrm{b}$ la pente de la droite de régression propre au prélèvement.

\subsection{Résultats}

\subsection{Niveau de population au début de chaque cycle biologique}

La population initiale d'œufs par arbre (X), représentée sur la figure 2, avec les estimations du nombre de chenilles présentes à chaque prélèvement $\left(\bar{y}^{\prime}\right)$ dans tous les biotopes, est élevée, dans l'ensemble, en 1965-1966. Pour le cycle 1967-1968, on observe une diminution de la population d'œufs dans les parcelles de basse altitude, 
et un accroissement dans certains sites d'altitude tels que Castellacce-Bas. Ce phénomène s'explique en partie par la mortalité de 1965-1966 mais peut également correspondre à un déplacement des adultes issus d’altitudes basses vers les zones d'altitude moins défeuillées. Deux parcelles furent particulièrement surpeuplées : Pente des Genêts en 1965 et Castellacce-Bas en 1967.

Du cycle 1967-1968 au cycle 1969-1970, la réduction du nombre d'œufs par pin se généralise à l'exception de Castellacce-Haut, dans toutes les parcelles étudiées.

La parcelle Mezza-Luna reste constamment caractérisée par un faible niveau de population.

\subsection{Nombre moyen d'xuls par ponte}

Chaque manchon de ponte étant déposé par une seule et même femelle, la moyenne du nombre d'oufs par ponte, dans chaque parcelle (figure 1), constitue une estimation de la fécondité des femelles ayant pondı. Cette moyenne évolue peu d'une année à lautre et se maintient autour de 200 œufs. Il est donc peu vraisemblable que la fécondité ait constitué, de 1965 à 1970, un facteur déterminant de l'évolution des populations. On observe, cependant, des variations pouvant avoir une signification biologique précise; en particulier :

- une certaine réduction de la fécondité en 1967, après le cycle 1965-1966, où les processionnaires ont soulfert de surpopulation et de virose;

- un nombre d'oufs élevé (205 à 230 aufs) dans les parcelles situées entre $1300 \mathrm{ct} 1400 \mathrm{~m}$ peut être lié au déplacement ae populations d'adultes des zones basses surpeuplées vers les parcelles d'altitude; l'existence de femelles dynamiques assurant lc maintien et la propagation de l'infestation est bien connue chez certaines espèces telles Malacosoma pliviale (Weldington, 1964, 1965).

\subsection{Mortalité au stade cuf}

La mortalité des oufs représente 10 à 15 p. 100 de la population en moyenne (fig. 1 et 2). Elle esí essentiellement due aux parasites et varie très peu d'année en annće. Le pourcentage d'individus parasites n'est que faiblement affecté par les variations numériques de lhôte; il s’accroît malgré tout légèrement en 1969, lors de la diminution des populations de processionnaires. Des différences importantes sont par contre notées d'un biotope à l'autre. Le parasitisme est plus faible dans les parcelles d'altitude exposées au Nord (Mezza-Luna); Ooencyrtus pityocampa Mercet n'est jamais très abondant; Tetrastichus servadei Dom. assez efficace dans les parcelles de basse altitude, a une incidence moindre dans les parcelles situées à plus de $1300 \mathrm{~m}$ (Castellacce-Bas, Castellacce-Haut), où l'on enregistre au contraire une action accrue des trichogrammes.

La stabilité du taux de mortalité des oufs ne permet pas d'accorder à ee facteur une action décisive dans l'évolution des populations au cours de ces trois cycles.

\subsection{Mortalité larvaire}

Les résultats concernant la réduction des populations larvaires au cours de chaque cycle sont résumées sur la figure 2 et sur la figure 3 qui montre parallèlement l'évolution de la régression entre le nombre de chenilles et le nombre d'œufs initialement déposés 
sur les pins échantillonnés. Sur le tableau 1 sont données simultanément par années et par parcelles les valeurs du nombre moyen de chenilles ( $Y$ ) et de la pente de la droite de régression (b) et sont présentés les différents groupes de régression mis en évidence par l'analyse statistique et les prélèvements entre lesquels les diminutions de population les plus importantes sont enregistrées.

Il existe une certaine analogie des phénomènes observés pour les différents cycles, dans chaque biotope. Certaines parcelles ensoleillées de basse altitude telles que PK 51 montrent une évolution très progressive de la mortalité et de la régression d'un stade à l'autre, alors que les parcelles soumises à des conditions plus rigoureuses d'altitude ou d'exposition comme Castellacce-Haut ou Mezza-Luna présentent des paliers plus accentués suivis de chutes importantes de la population, et d'un abaissement brutal de la pente de la droite de régression.

La mortalité affectant les premiers âges larvaires est dans tous les cas faible et ne paraît pas avoir, dans le Niolo, une incidence déterminante (des observations identiques sont faites par G. Demolin, au Mont Ventoux).

En revanche, la mortalité affecte essentiellement les stades suivants, à partir du troisième, mais son importance même et les phases critiques auxquelles elle intervient varient selon les trois cycles. Ces différences ne peuvent s'expliquer par l'impact des parasites.

En effet, le parasitisme des larves essentiellement dû à Phryxe caudata Rond. présente également, comme le parasitisme des oufs, des variations relativement faibles. Toutefois (fig. 1), on observe, en 1969, lorsque les populations de processionnaire régressent, un taux de parasitisme plus élevé (cette évaluation étant faite en période préhivernale, époque à laquelle les données sont les plus représentatives de l'action de ce parasite).

Par contre, les variations de populations šinterprètent assez simplement si l'on tient compte des conditions climatiques et de leur incidence sur la phénologie de l'insecte, ainsi que du niveau de population initial de chacune des générations.

Nous disposons pour cela des informations climatiques (figure 4) du poste de la Météorologie Nationale implanté à la maison forestière de Popaja dans une situation altitudinale moyenne $(1074 \mathrm{~m})$ du massif forestier et des observations faites régulièrement sur l'évolution dans le temps des colonies de chenilles sur un ensemble de pins voisins, dans chaque parcelle, des pins échantillonnés. Ces observations sont rapportées sur la figure 5, où les segments de droites qui séparent les stades correspondent aux éclosions, aux mues et à la dispersion et donnent schématiquement le pourcentage des individus qui ont subi ces événements.

\subsection{Cycle 1965-1966}

Le cycle 1965-1966 durant lequel les chenilles ont évolué sous des conditions climatiques favorables peut être pris comme référence. Les dates d'éclosion sont d'autant plus tardives que l'altitude est élevée. Cet écart s'accroît au cours des premiers stades et on note que la population de Mezza-Luna, en exposition Nord, se développe plus lentement que celle de Pente des Genêts, située plus haut, mais mieux exposée. Duns les parcelles les plus favorisées (PK 51 et Pente des Genêts), il y a quelques larves du $5^{\text {e }}$ stade avant l'hiver; au contraire, à Castellacce-Haut, de nombreuses 

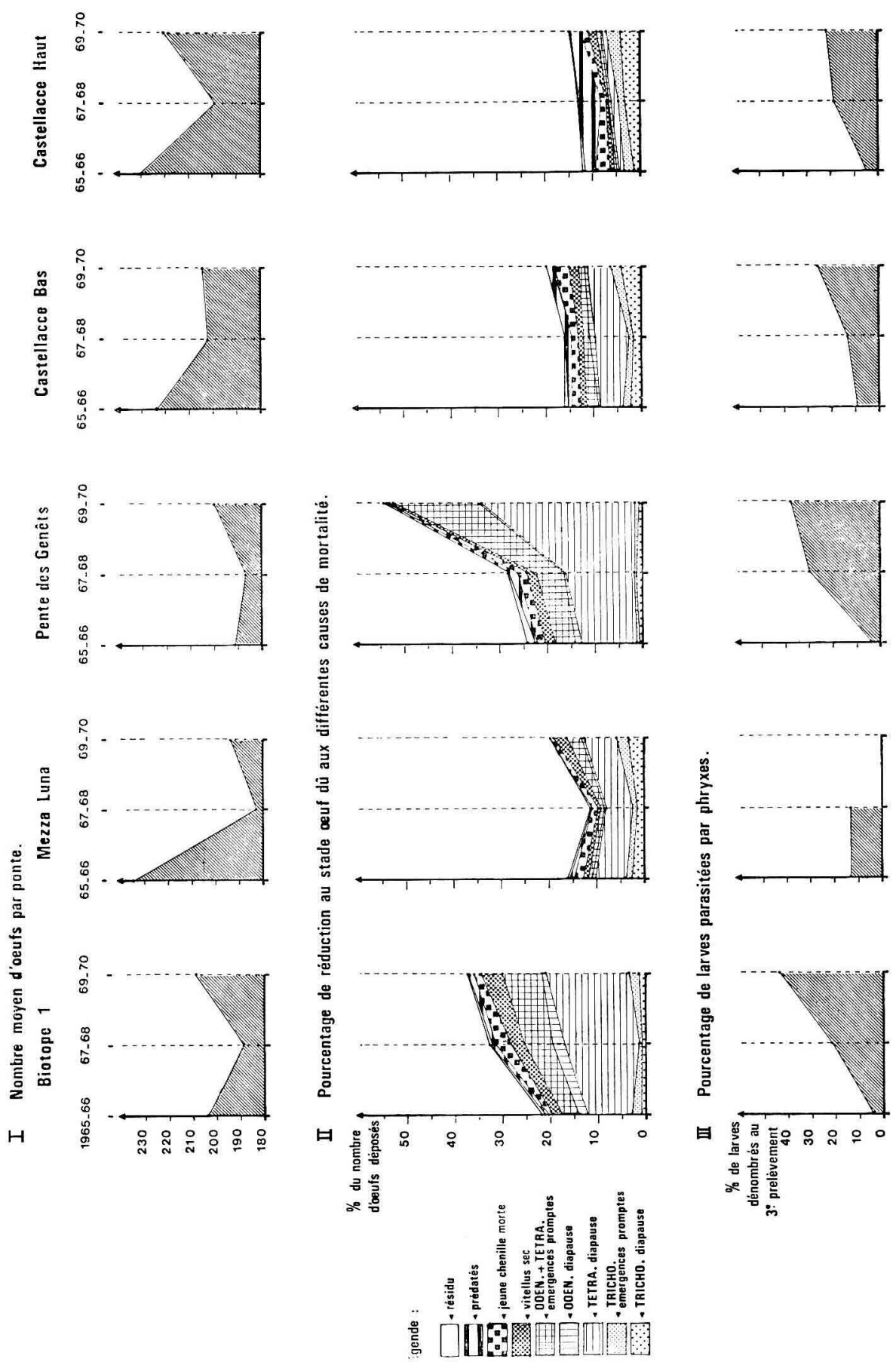

FIG. 1

Evolution du nombre moyen d'œufs par ponte, de la mortalité au stade œuf et du parasitisme des larves par Phryxe caudata Rond.

Evolution of egg mean number for egglaying, of egg mortality and larval parasitism by Phryxe caudata Rond. 
chenilles ne se trouvent qu'au $3^{\circ}$ stade. Ces cas extrêmes mis à part, ce sont les larves du $4^{\mathrm{e}}$ stade qui subissent la période hivernale. Au printemps, la reprise d'activité est rapide et les derniers stades sont d'autant plus courts que le développement antérieur a été long. La dispersion des chenilles en procession a lieu en mai où les différences précédemment observées sont encore marquées mais suffisamment faibles pour que la quasi-totalité de cette dispersion s'effectue dans toutes les parcelles en un seul mois.

La mortalité se manifeste surtout entre le $3^{\circ}$ et le $4^{\prime \prime}$ prélèvement, au troisième stade larvaire essentiellement, avec une tendance à affecter des stades plus jeunes en altitude (Castellacce-Haut, 2" stade) et plus âgés dans les localisations peu élevées (PK 51, $4^{*}$ stade). Elle est essentiellement préhivernale et particulièrement élevée dans les parcelles surpeuplées (Pente des Genêts, Castellacce-Bas) où on enregistre des phénomènes de famine caractérisés. Cette réduction coïncide dans les régions de basse altitude avec la manifestation épidémique d'une virose à Smithiavirus pityocampae Vago (PK 51, Pente des Genêts), mais est enregistrée dans toutes les parcelles et résulte vraisemblablement du haut niveau de population atteint en 1965 dans tout le massif forestier. La mortalité hivernale est par contre extrêmement faible et coïncide avec un hiver doux.

\subsection{Cycle 1967-1968}

Par rapport à l'hiver 1965-1966, l'hiver 1967-1968 présente des températures moyennes plus basses et une durée d'enneigement accrue. En ce qui concerne les températures estivales et automnales, 1967 est un peu plus chaud que 1965, ce qui peut s'expliquer par l'abondance des précipitations de 1965 (cf. courbes pluies et nébulosité). Les pontes et les éclosions présentent d'une parcelle à l'autre, un décalage dans le temps identique à celui du cycle 1965-1966. La durée des premiers stades est comparable mais leur développement est plus lent dans les parcelles de basse altitude (PK 51) et plus rapide dans les parcelles élevées, de telle sorte que Castellacce-Bas est en décembre la parcelle la plus avancée et la seule à présenter des chenilles en L 5, ce que peut expliquer l'ensoleillement plus important d'été et d'automne par rapport au cycle précédent. A cette exception près, les chenilles passent l'hiver en $\mathrm{L} 4$, le développement hivernal est plus lent qu'en 1965-1966, vraisemblablement à cause de l'hiver plus rigoureux et se traduit malgré un $5^{\text {e }}$ stade plus bref par des processions plus tardives au printemps suivant.

La mortalité est plus tardive; on l'enregistre principalement entre les prélèvements 4 et 5 , c'est-à-dire sur le $4^{\prime \prime}$ stade. Toutefois, certaines parcelles très peuplées, comme Castellacce-Bas, présentent encore une mortalité du type de 1965-1966, avec des phénomènes de jeûne caractérisés et une chute de population préhivernale au $3^{\text {e }}$ stade larvaire; des cas de viroses sont toujours notés à Pente des Genêts. Mais ce cycle est surtout caractérisé par une mortalité hivernale due à un hiver froid, d'autant plus importante que les parcelles se trouvent en exposition nord ou en altitude élevée.

\subsection{Cycle 1969-1970}

Le déficit thermique, par rapport au cycle 1965-1966, est encore plus accentué pour l'hiver 1969-1970. Celui-ci, particulièrement froid et précoce est précédé par un mois d'octobre médiocre et se prolonge au printemps. L'enneigement atteint sa durée maximum et la neige reste pratiquement en place de fin novembre à fin mars. 


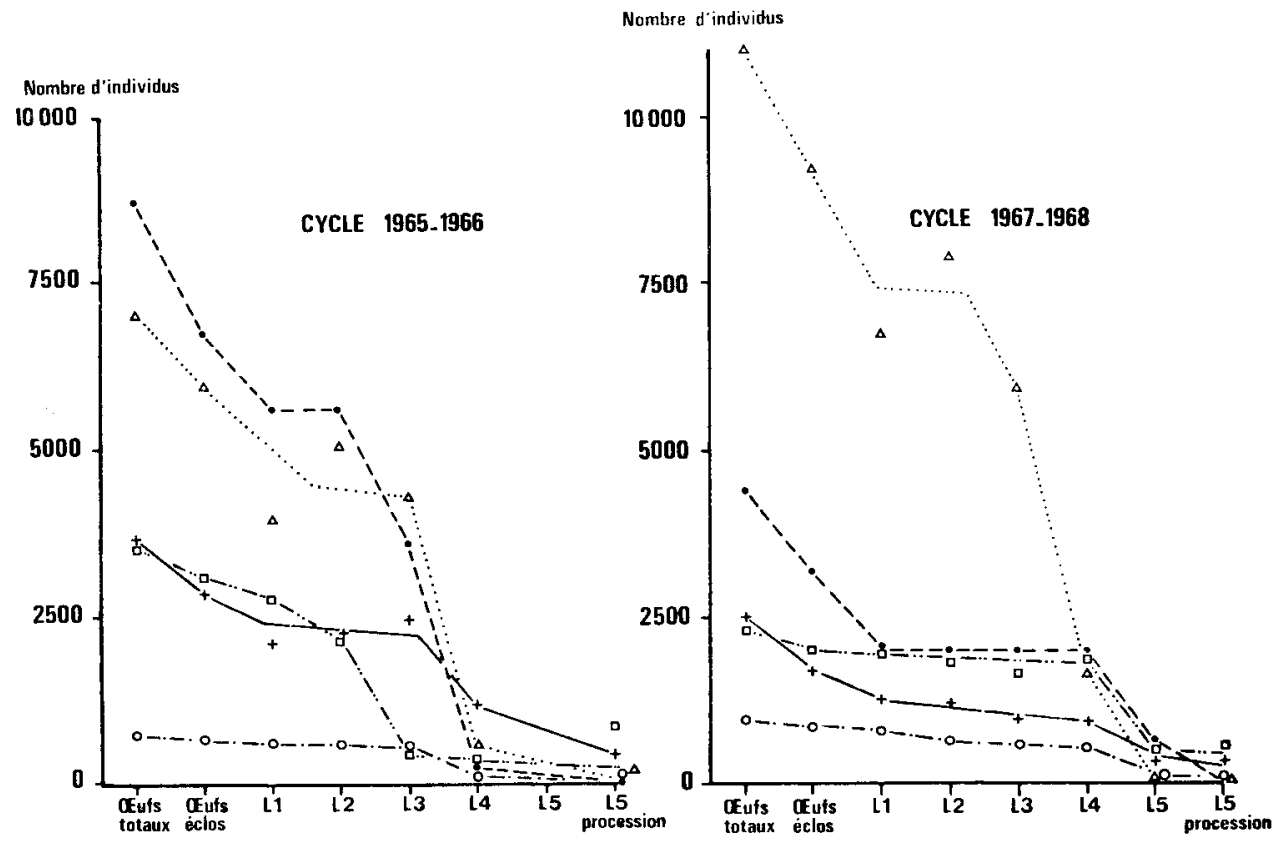

Nombre d'individus

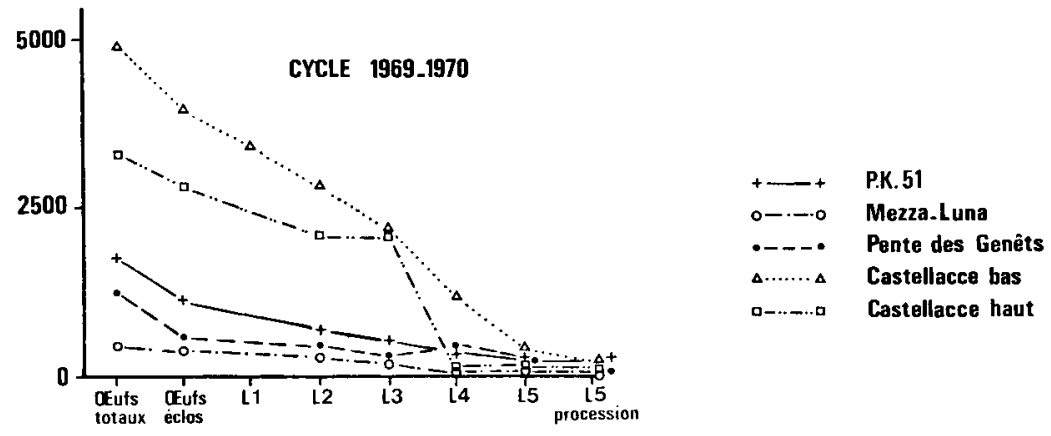

FIG. 2

Evolution du nombre moyen d'individus présents sur les pins au cours des cycles étudiés.

Evolution of mean population on pines during studied life cycles.

L'évolution est particulièrement lente : la lenteur de l'incubation et du développement des premiers stades est liée à des dépôts de ponte tardifs et aux faibles températures du mois de juillet et de l'automne. Si les parcelles de basse altitude (PK 51) parviennent au $4^{\circ}$ stade avant l'hiver, une proportion de larves, d'autant plus forte que le site se trouve plus en altitude ou en exposition nord, aborde l'hiver, au $3^{\circ}$ stade, ou en cours de mue. Castellacce-Haut et Mezza-Luna sont les parcelles les plus défavorisées. L'évolution hivernale est très ralentie et la reprise d'activité 


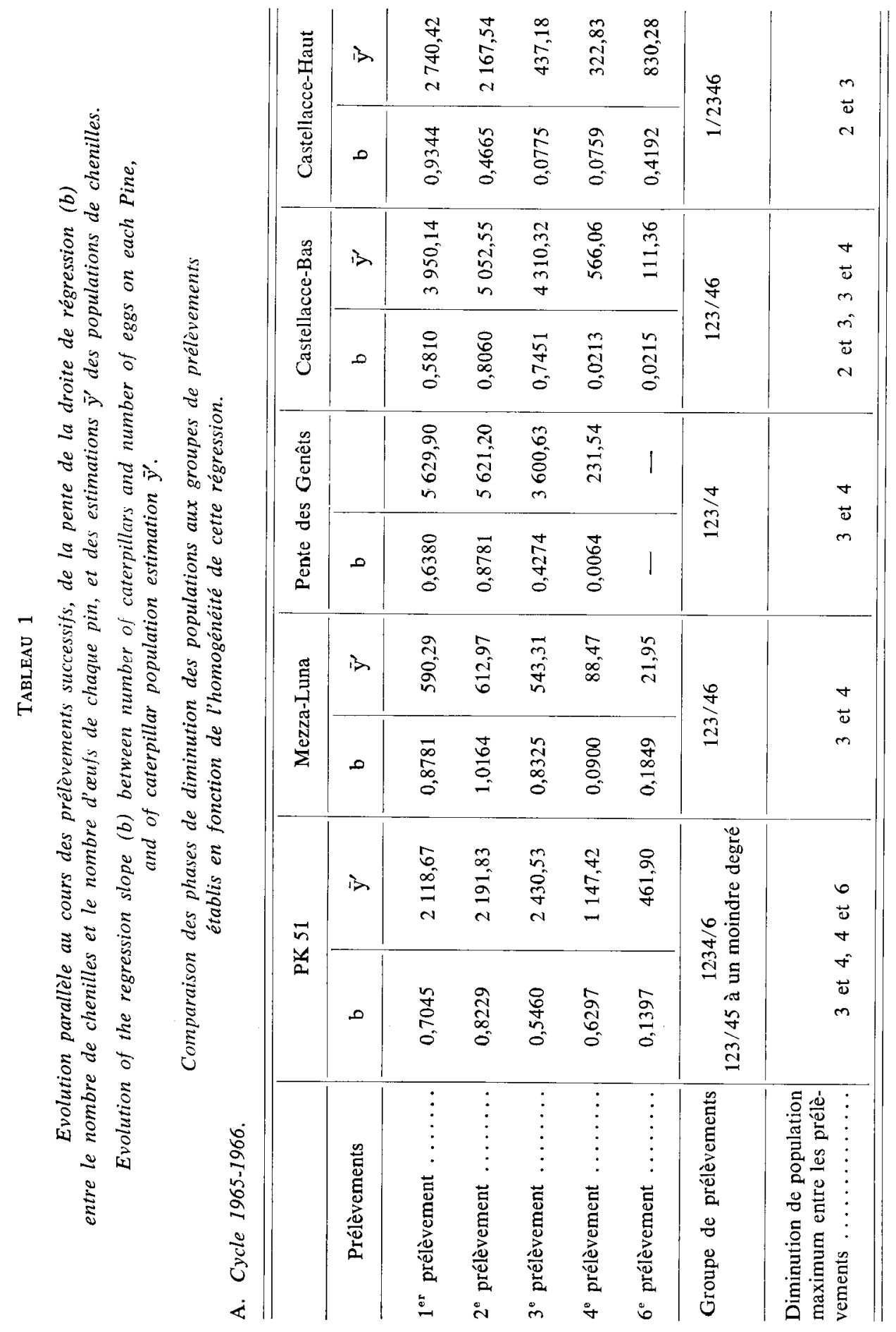



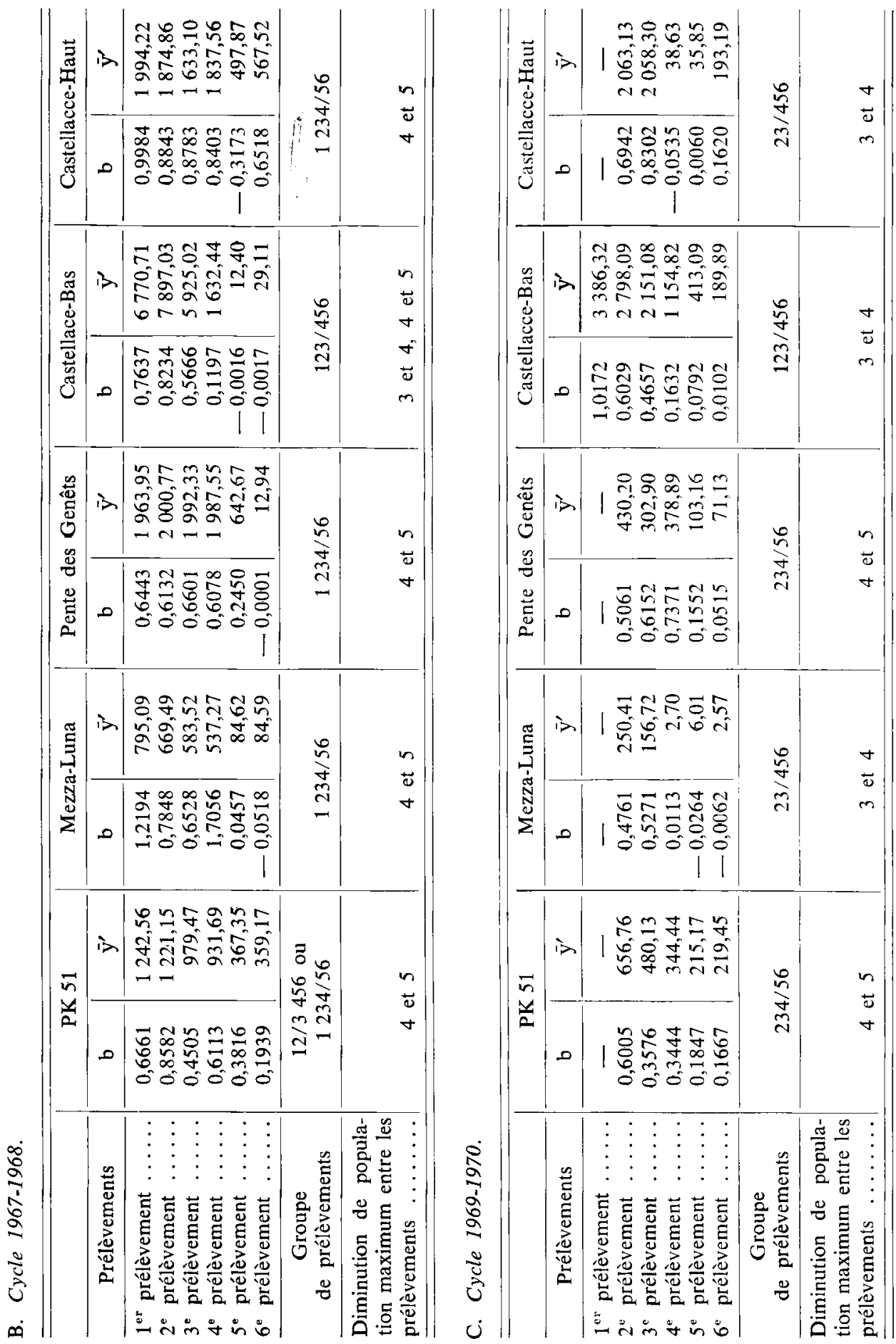
tardive à cause de la prolongation de l'hiver. Malgré un $5^{\circ}$ stade bref, les processions ont lieu encore plus tard qu'en 1968 et ne commencent pas avant la fin mai.

P.K. 51

MEZZA LUNA

PENTE DES GENETS

CASJELLACCE BAS

CASTELLACCE HAUT
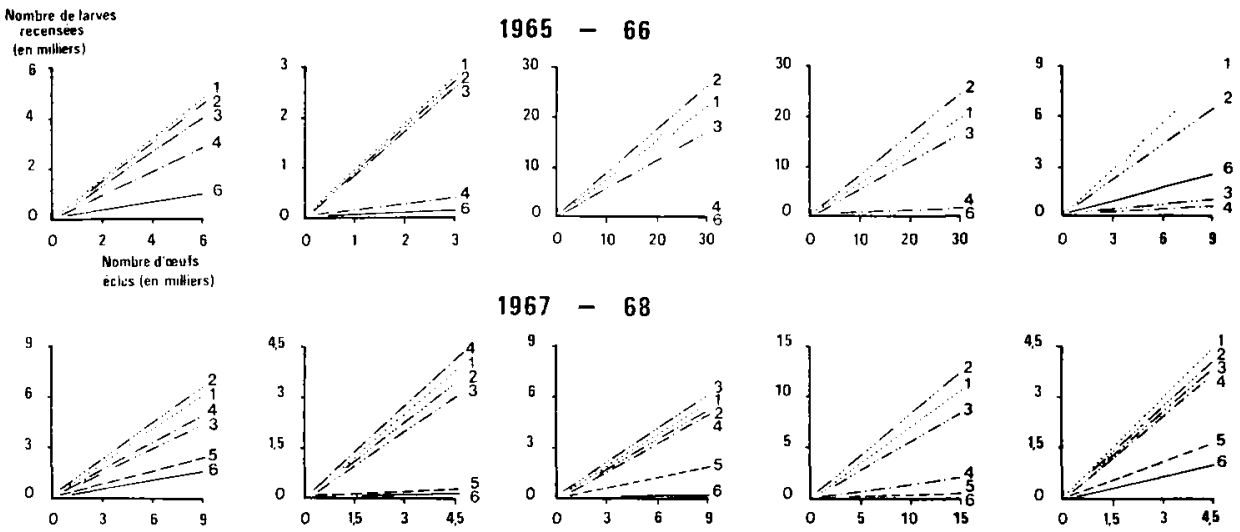

$1967-68$
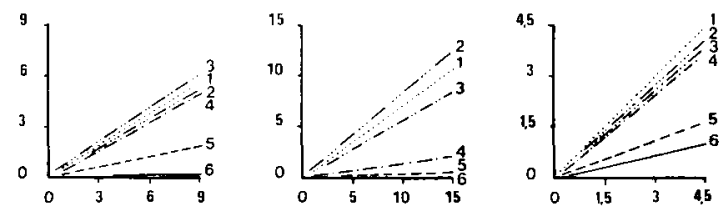

$1969-70$
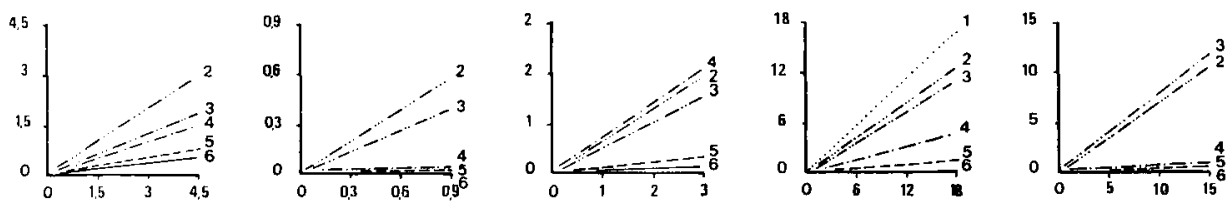

LEGENDE: 1,2,3,4,5 Prélèvements aux différents stades larvaires

6 . " avant la dieperaion

FIG. 3

Evolution en fonction des différents prélèvements de la régression entre le nombre de larves et la population initiale d'wufs des arbres.

Relationships between larval number and egg initial population on trees at different records.

La mortalité est principalement hivernale, elle se produit avec son maximum d'intensité entre le $4^{\circ}$ et le $5^{\mathrm{e}}$ prélèvement $\left(4^{\mathrm{e}}\right.$ stade $)$ dans les parcelles de basse altitude (PK 51, Pente des Genêts), et entre le $3^{\mathrm{e}}$ et $4^{\circ}$ prélèvement $\left(3^{\circ}\right.$ stade) dans les sites élevés ou en exposition Nord (Castellacce, Mezza-Luna). Elle est d'autant plus accentuée (figures 2 et 3 ) que les parcelles sont situées à une altitude élevée ou en exposition Nord (Castellacce-Haut, Mezza-Luna). Les observations sur le terrain confirment ce fait : à cause de la lenteur du développement larvaire et de la précocité de l'hiver, de nombreuses chenilles, surtout dans les parcelles où elles étaient défavorisées par lexposition et l'altitude, étaient encore au $3^{\mathbf{e}}$ stade, ou à la mue $\mathbf{L} 3-\mathrm{L} 4$, lors des premières chutes de neige, fin novembre, et n'avaient pas tissé de nids définitifs suffisamment structurés pour affronter la mauvaise saison. Beaucoup moururent durant l'hiver. Celui-ci fut très rigoureux et le recouvrement des arbres par la neige ne permit 
que de rares prises de nourriture. De plus, toutes les colonies situées sous la couche de neige ( $7 \mathrm{~m}$ à Castellacce) périrent. Enfin, à la reprise d'activité, au printemps, les dernières chenilles qui n'avaient pas atteint le $4^{\circ}$ stade, furent incapables de reprendre leur tissage et moururent.
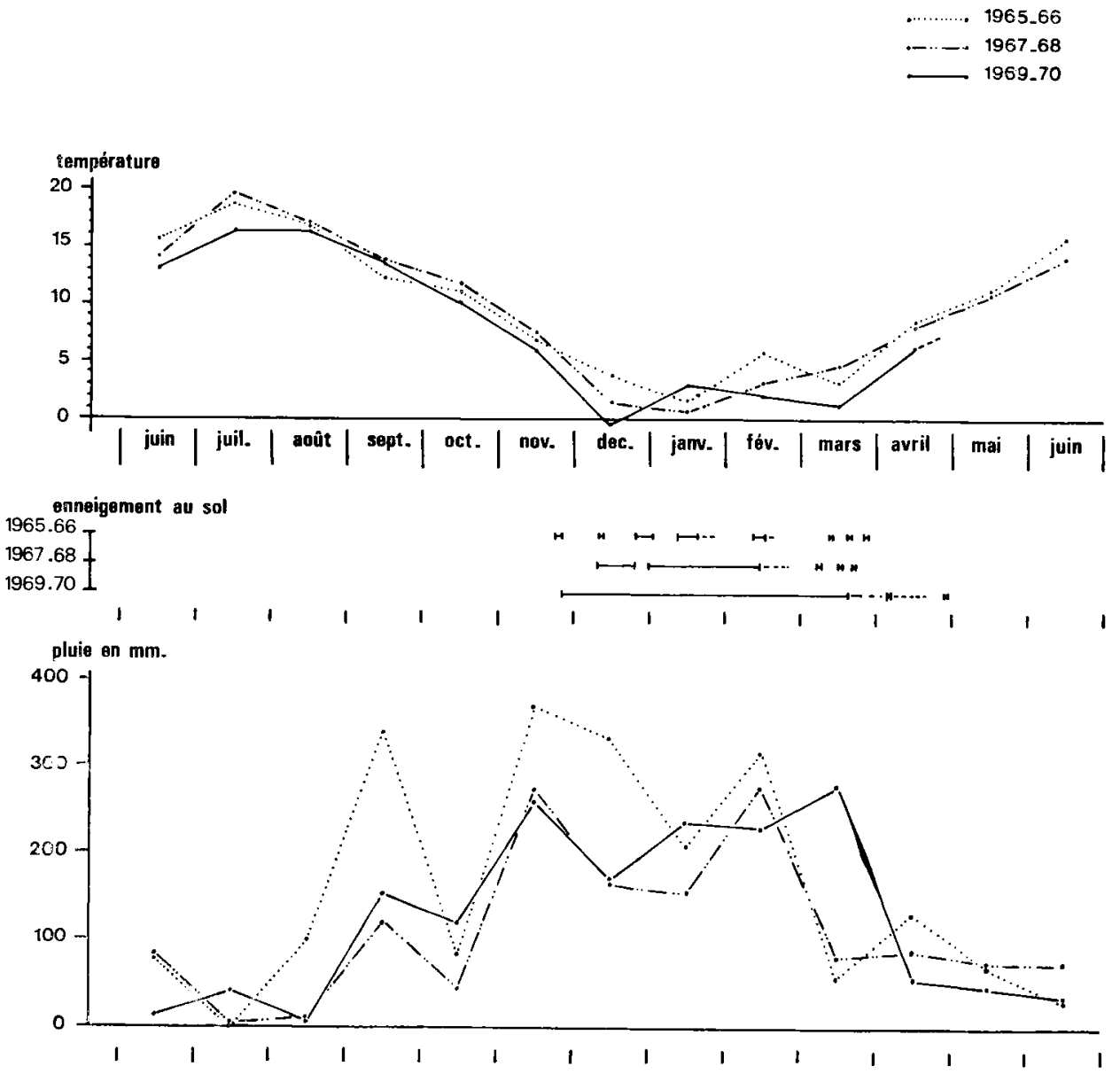

\section{nóbulosité de $\mathbf{0}$ à 8}

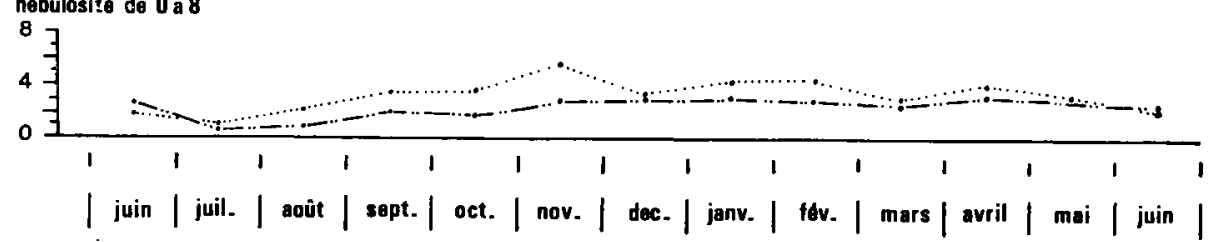

FIG. 4

Observations climatologiques effectuées de 1965 à 1970 à la Maison forestière de Popaja. Meteorological data from 1965 to 1970 a: the "Maison forestière de Popaja». 
I CYCLE 1965-1966

P.K. 51

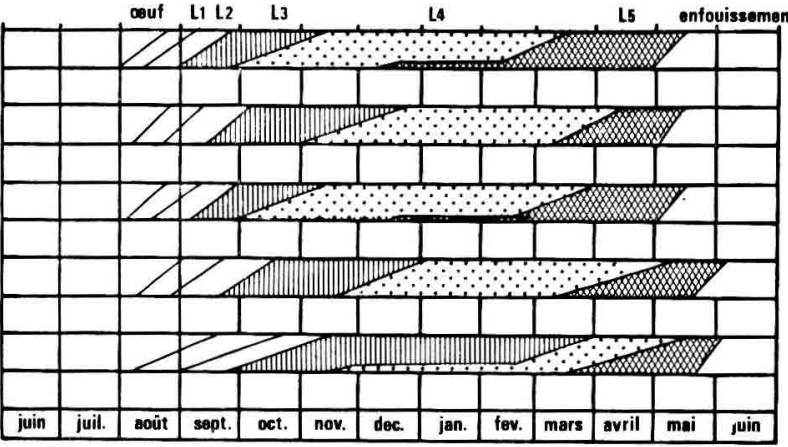

II CYCLE 1967_1968

Températures

mensuelles

moyennes

Popaja (1074 m.)

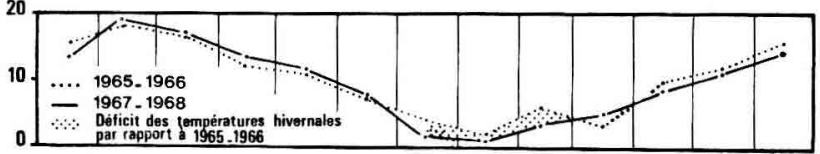

P.K. 51

MEZZA - LUNA

PENTE DES GENETS

CAstellacce bas

CAstellacce haut
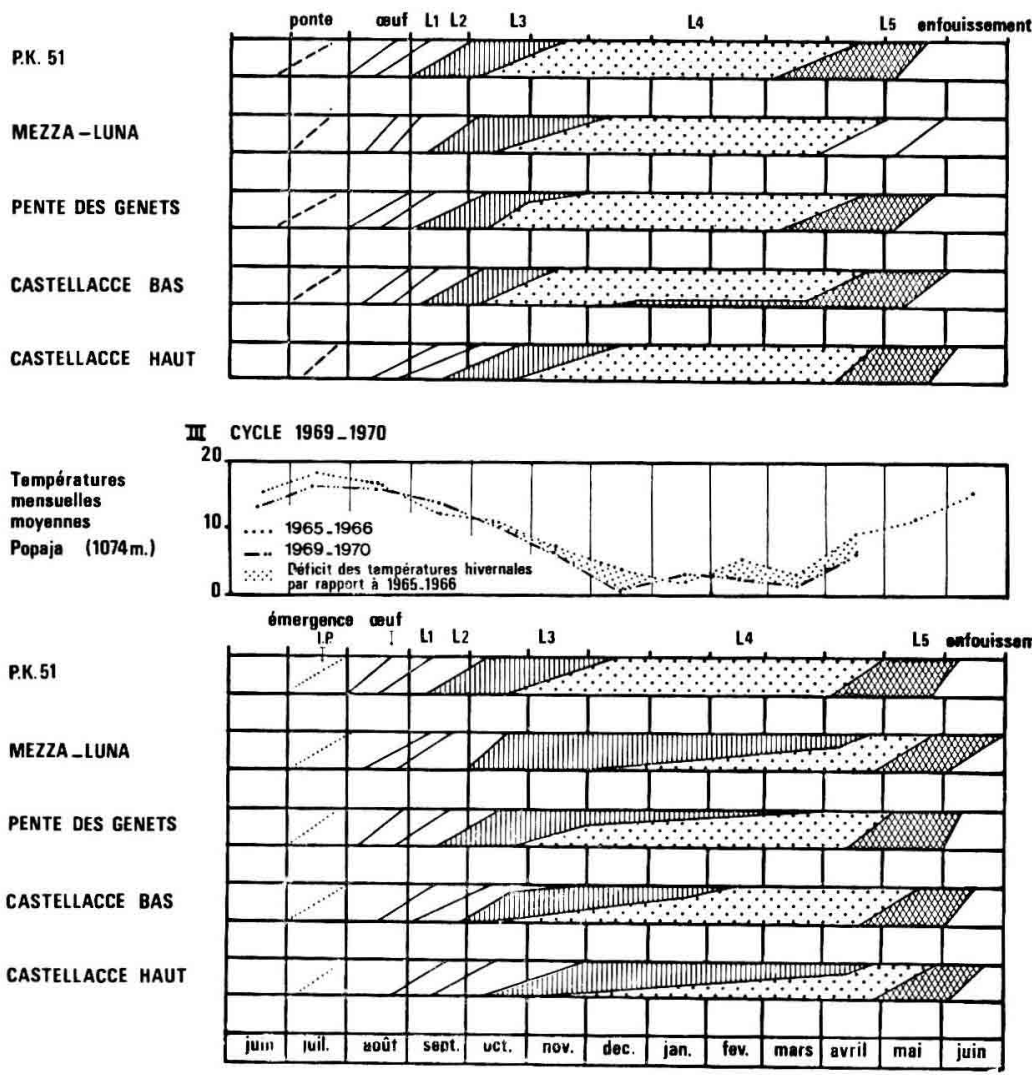

FIG. 5

Phénologie de la processionnaire durant les trois cycles étudiés.

Pine Processionnary phenology during the three studied life cycles. 
CYCLE 1965.1966
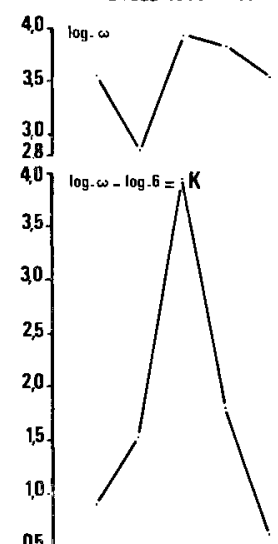

0,5

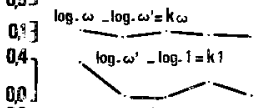

Q2. $\log 1-\log -2 \times k 2$

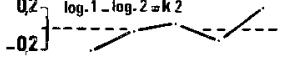

Q,5 $\log .2-\log -3=k 3$
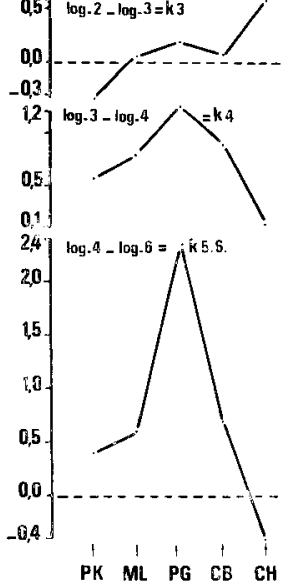

Légende

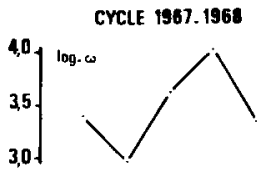

CYCLE 1969.1970
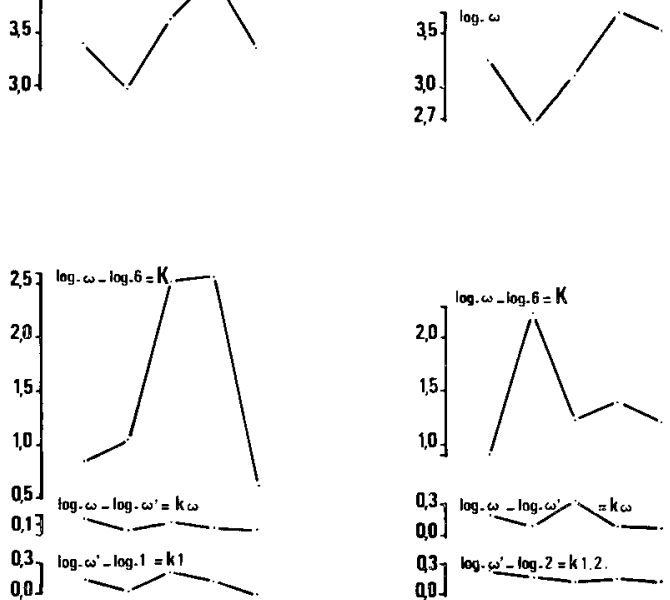

$0.21 \log .1-\log .2=k 2$

$0,2][-2=$
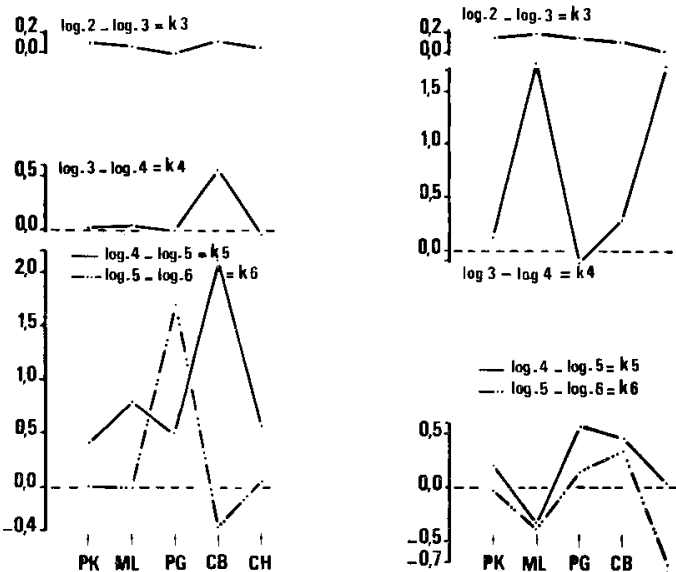

$-\log -4-\log -5=k 5$

$-. . \log .5-\log .6=k 6$

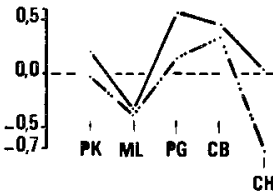

$\omega$; nombre d'oeufs déposés

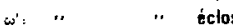

1 nombre de chenilles des stades 1

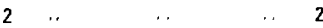

$3 \quad$.. $\quad$.. $\quad$.. 3

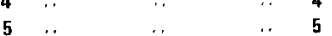

6- nombre de chenilles avant processions

FIG. 6

Etude comparative de la mortalité totale des processionnaires durant leur vie sur l'arbre $(K)$ et de la mortalité à chaque stade $(k)$ suivant la méthode de Varley \& Gradwell.

Comparative study between total Processionnary mortality during its development on tree $(K)$ and mortality at each stage $(k)$ following the Varley's \& Gradwell's method. 


\subsection{Discussion}

Il est intéressant de constater que la processionnaire, insecte généralement astreint, pour une grande part, à une vie larvaire active durant l'hiver, et passant pour y être bien adapté (Demolin, 1969) semble cependant se trouver souvent, dans les vallées d'altitudes corses, dans cies conditions climatiques limitantes.

Durant les trois cycles étudiés dans le Niolo, les agents biotiques (parasites, virus) ne paraissent pas avoir eu un rôle déterminant; la mortalité au cours de la vie aérienne a été principalement conditionnée par le niveau même de population atteint et par des facteurs climatiques déterminant durant l'hiver une mortalité larvaire d'autant plus élevée que les parcelles sont exposées au Nord ou situées plus en altitude et que le développement est plus tardif.

Ces différentes conclusions sont illustrées de façon un peu différente par une représentation graphique empruntée à VARLET \& GradWell (1960) (fig. 6); sur cette figure, les variations de la mortalité d'un biotope à l'autre, sont représentées comparativement pour chaque stade et pour l'ensemble de la vie larvaire.

Le niveau de population est exprimé en logarithmes.

La mortalité entre deux prélèvements est traduite par le logarithme du niveau de population du premier prélèvement (A) moins le logarithme du niveau de population du prélèvement suivant (B), soit :

$$
\mathbf{k}=\log \mathrm{A}-\log \mathrm{B}=\log \frac{\mathrm{A}}{\mathrm{B}},
$$

ce rapport étant d'autant plus élevé que la mortalité est forte entre les deux prélèvements.

De même, la mortalité totale du cycle peut s'exprimer par :

$$
\mathrm{K}=\log (\omega)-\log (6),
$$

où $(\omega)$ représente la population au stade cufs et $(6)$ celle du $6^{\mathrm{e}}$ prélèvement (dispersion).

Elle est la somme des mortalités $\mathrm{k}$, observées à chaque stade, entre deux prélèvements.

La similitude des variations de la mortalité totale $\mathrm{K}$ et de la mortalité relative à un stade déterminé $\mathrm{k}$ montre l'importance de la mortalité à ce stade pour rendre compte des différences de mortalité observées sur l'ensemble de la vie larvaire d'une parcelle à l'autre.

Bien que la mortalité au stade ouf soit notable, ses variations sont sans rapport avec celles de la mortalité totale. Il en est de même pour les premiers stades larvaires.

Par contre, la mortalité au cours des derniers stades a beaucoup d'importance :

- en 1965-1966, la courbe entre le $3^{\mathrm{e}}$ et le $4^{\mathrm{e}}$ prélèvement $\left(3^{\mathrm{e}}\right.$ stade larvaire) évoque déjà celle de la mortalité totale et met en évidence l'effet de la mortalité de la parcelle la plus surpeuplée (Pente des Genêts). Le phénomène est encore plus net pour les derniers stades larvaires;

- en 1967-1968, on voit apparaître de la même façon dès le $3^{\circ}$ stade, l'incidence de la mortalité de la parcelle surpeuplée Castellacce-Bas, qui se poursuit comme 
dans le cas précédent au cours du stade suivant : la mortalité survenant entre les prélèvements 4 et 6 est étroitement liée à la mortalité totale ;

— en 1969-1970, la mortalité intervient de façon déterminante au $3^{\text {e }}$ stade pour les parcelles les plus défavorisées par les conditions climatiques et d'une façon moindre au $4^{\mathrm{e}}$ stade pour les sites les plus ensoleillés;

- enfin, si les courbes de mortalité totale des cycles 1965-1966, et 1967-1968, présentent une certaine analogie avec les courbes du niveau de population, cette relation disparaît totalement en 1969-1970.

\section{Etude des fluctuations transcycliques}

\subsection{Méthodes}

Les nids de processionnaire sont recensés, après l'hiver pour chaque cycle, sur 1500 pins environ, répartis sur toute la vallée selon un échantillonnage au hasard stratifié (C. Geri, 1971). Dans le double but d'améliorer la précision statistique des résultats et de contrôler localement l'évolution des populations, le massif est divisé en 10 zones et 34 sous-zones. Les zones sont délimitées en fonction des grandes divisions topographiques de la vallée, les sous-zones correpondant aux principaux types de peuplements qui forment des étages altitudinaux : peuplements clairs de basse altitude, denses aux environs de $1000 \mathrm{~m}$, moyennement denses de 1200 à $1350 \mathrm{~m}$ en moyenne, pins isolés tabulaires qui s'étendent jusqu'aux limites altitudinales (1 500 $1600 \mathrm{~m})$ de la forêt ${ }^{(*)}$.

Dans chaque sous-zone des placettes de $400 \mathrm{~m}^{2}$ sont préalablement tirées au hasard sur carte à chaque cycle. Les dénombrements sont effectués à raison d'un pin par placette. En 1970, la précision constatée a permis, sans grand risque, de diminuer l'intensité des sondages : les dénombrements furent effectués à raison d'une placette pour 5 hectares au lieu de 4 hectares, au cours des cycles précédents; toutefois dans les zones $\mathrm{E}, \mathrm{F}$ et $\mathrm{H}$ un plan de sondage hiérarchisé fut réalisé afin de déterminer le meilleur mode d'échantillonnage; celui-ci n'a pas apporté de résultats permettant de justifier, compte tenu des impératifs pratiques, une modification du plan de sondage initial.

Les estimations de la variance des populations sont données :

- au niveau de la sous-zone par la formule proposée par C. Millier, Directeur de la Station de Biométrie de l'I.N.R.A. à Nancy, $\operatorname{Var} N \overline{\mathrm{x}}=\mathrm{N}^{2} \operatorname{Var} \overline{\mathrm{x}}+\overline{\mathrm{x}}^{2} \operatorname{Var} \mathrm{N}$ $+2 \overline{\mathrm{x}} \mathrm{N}(\operatorname{cov} . \overline{\mathrm{x}} \mathrm{N})$, où $\overline{\mathrm{x}}$ est le nombre moyen de nids par pin, $\mathrm{N}$ l'estimation du nombre total de pins dans la sous-zone;

- au niveau de la zone, par la somme des estimations des sous-zones;

- au niveau des étages de peuplement et de la vallée, par la somme des estimations des zones.

Le terme $2 \overrightarrow{\mathrm{x}} \mathrm{N}(\operatorname{cov} \overrightarrow{\mathrm{x}} \mathrm{N}$ ) est négligé. Cette covariance est généralement négative. Il en résulte que l'erreur est un peu surestimée.

(*) On dénombre environ 400 pins à l'hectare en moyenne pour les peuplements denses, 200 pour les moyennement denses et 80 pour les deux étages de pins clairs. 
Les dénombrements sont complétés par une collecte de nids pour examens parasitologiques et pathologiques, à raison d'un pour 5 placettes environ; 15 chenilles par nid sont disséquées pour l'observation des parasites et servent à la confection de frottis pour l'examen des maladies. Ces informations ne traduisent en fait l'état sanitaire des populations qu'au seul $5^{\circ}$ âge larvaire. Elles fournissent cependant des images de l'activité des facteurs biotiques représentatives de toute la vallée et comparables entre cycles.

Enfin, sont relevées certaines caractéristiques de l'environnement forestier sur toutes les placettes échantillonnées :

- au niveau des placettes:

- l'exposition ;

- l'altitude ;

- la nature des peuplements (nombre d'étages de pins laricio, hauteur moyenne et densité des arbres, mélange avec des feuillus);

- le nombre de pins.

- au niveau de l'arbre échantillonné :

- la hauteur et le diamètre à $1 \mathrm{~m} 30$.

Ces données, outre leur intérêt pour la connaissance des facteurs de variabilité du niveau de population et l'amélioration du plan d'échantillonnage, sont utiles pour interpréter la répartition des populations ainsi que les fluctuations enregistrées d'un cycle à l'autre. Le nombre de pins intervient dans les calculs de population.

\subsection{Evolution des populations}

\subsection{Résultats}

Les estimations des niveaux de population à chaque cycle, par zones, par souszones, par types de peuplement et pour toute la vallée sont rapportées (tabl. 2). Les nombres moyens de nids par pins observés chaque année dans les différentes souszones sont également représentés sur la carte du Niolo (figure 7).

On enregistre, du cycle 1965-1966 au suivant, une réduction relativement faible mais significative du niveau de la population de la vallée, qui diminue de 8758000 nids en 1966 à 6822000 en 1968 (valeurs arrondies en milliers). Cette réduction affecte la quasi-totalité des zones et sous-zones à l'exception de quelques zones d'altitude $(\mathrm{D}, \mathrm{E}$ et $\mathrm{J})$ constatation qui recoupe l'hypothèse, suggérée par l'étude parcellaire, d'une colonisation ou d'un maintien préférentiel des populations dans les zones d'altitude. De même, la figure 7 montre une répartition très voisine des populations en 1966 et en 1968, mais une diminution dans la zone $F$ de basse altitude et un accroissement dans les peuplements d'altitude des zones $D$ et $E$, et des zones H, I, J qui forment la vallée annexe de Calasima.

Du cycle 1967-1968 au cycle 1969-1970, la réduction de population est beaucoup plus importante et généralisée dans toute la vallée. Le niveau de population 
TABleau 2

Estimations du nombre de nids de processionnaires par sous-zone, par zone, par étage de peuplement et au niveau de la vallée.

Estimations of number of processionnary nests by sub-zone, by zone, by forest story, and at the whole valley scale.

I. Cycle 1965-1966.

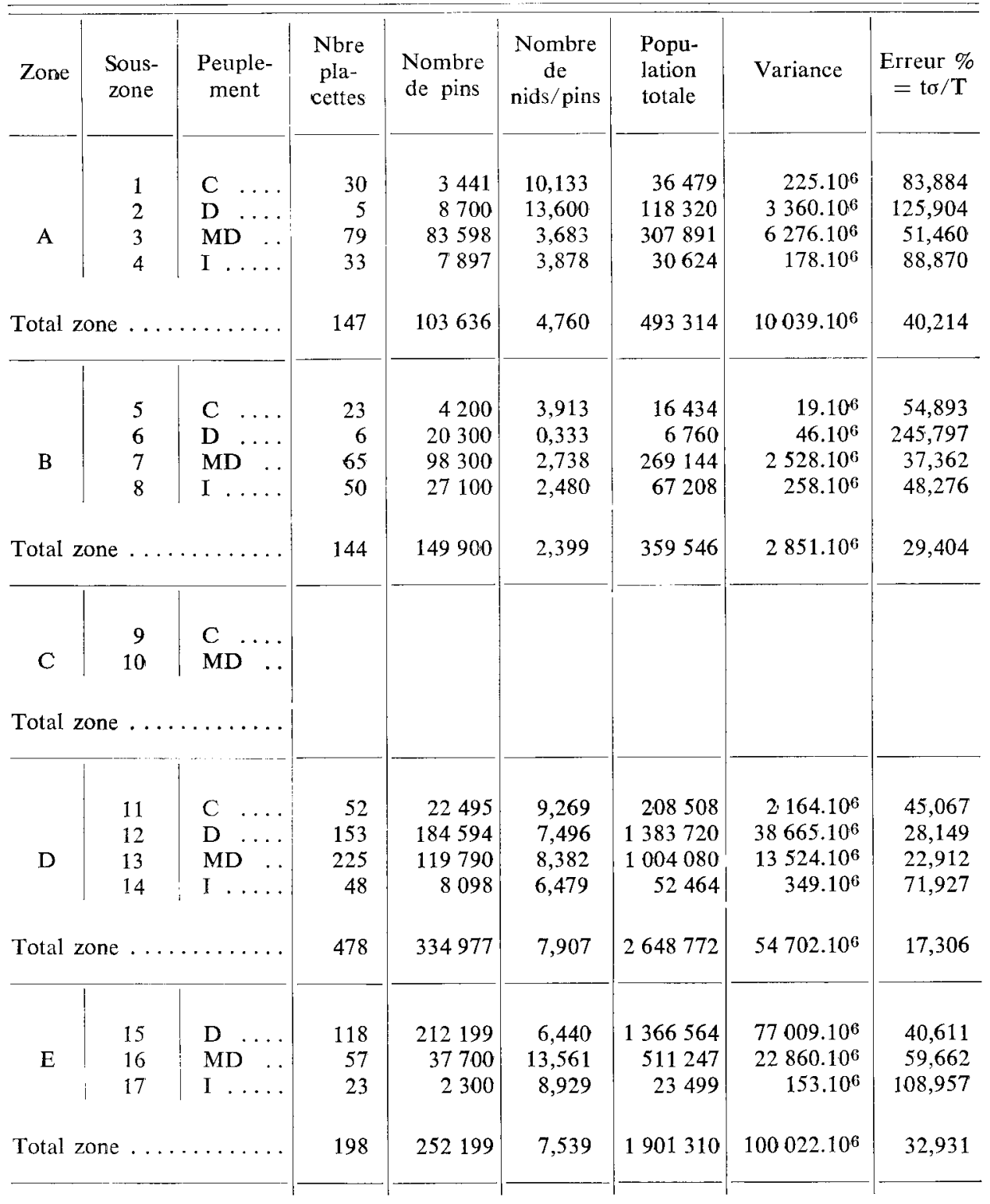




\begin{tabular}{|c|c|c|c|c|c|c|c|c|}
\hline Zone & $\begin{array}{l}\text { Sous- } \\
\text { zone }\end{array}$ & $\begin{array}{l}\text { Peuple- } \\
\text { ment }\end{array}$ & $\begin{array}{c}\text { Nbre } \\
\text { pla- } \\
\text { cettes }\end{array}$ & $\begin{array}{l}\text { Nombre } \\
\text { de pins }\end{array}$ & $\begin{array}{c}\text { Nombre } \\
\text { de } \\
\text { nids/pins }\end{array}$ & $\begin{array}{l}\text { Popu- } \\
\text { lation } \\
\text { totale }\end{array}$ & Variance & $\begin{array}{c}\text { Erreur } \% \\
=\mathrm{t} \sigma / \mathrm{T}\end{array}$ \\
\hline F & $\begin{array}{l}18 \\
19 \\
20\end{array}$ & $\begin{array}{l}\mathrm{C} \\
\mathrm{D}\end{array}$ & $\begin{array}{l}40 \\
23 \\
25\end{array}$ & $\begin{array}{l}19900 \\
18000 \\
15300\end{array}$ & $\begin{array}{r}8,000 \\
17,478 \\
13,000\end{array}$ & $\begin{array}{l}159200 \\
314600 \\
198900\end{array}$ & $\begin{array}{r}1125.10^{6} \\
13046.10^{6} \\
2966.10^{6}\end{array}$ & $\begin{array}{l}42,558 \\
75,141 \\
56,405\end{array}$ \\
\hline \multicolumn{3}{|c|}{ Total zone } & 88 & 53200 & 12,645 & 672700 & $17137.10^{6}$ & 38,799 \\
\hline G & $\begin{array}{l}21 \\
22 \\
23 \\
24\end{array}$ & $\begin{array}{l}\mathrm{C} \ldots \\
\mathrm{D} \ldots \\
\mathrm{MD} \\
\mathrm{I} \ldots\end{array}$ & $\begin{array}{l}21 \\
59 \\
32 \\
13\end{array}$ & $\begin{array}{r}14799 \\
116495 \\
25798 \\
5900\end{array}$ & $\begin{array}{r}12,571 \\
9,203 \\
16,562 \\
24,191\end{array}$ & $\begin{array}{r}186034 \\
1072 \\
0708 \\
427273 \\
141586\end{array}$ & $\begin{array}{r}2837.10^{6} \\
65095.10^{6} \\
20783.10^{6} \\
4671.10^{6}\end{array}$ & $\begin{array}{r}58,434 \\
48,074 \\
68,848 \\
104,265\end{array}$ \\
\hline \multicolumn{3}{|c|}{ Total zone } & 125 & 162992 & 11,209 & 18270.01 & $93386.10^{6}$ & 33,119 \\
\hline $\mathrm{H}$ & $\begin{array}{l}25 \\
26 \\
27\end{array}$ & $\begin{array}{ll}\mathrm{C} & \ldots \\
\mathrm{D} & \ldots \\
\mathrm{MD} & \cdots\end{array}$ & $\begin{array}{l}47 \\
14 \\
15\end{array}$ & $\begin{array}{l}15797 \\
12099 \\
20700\end{array}$ & $\begin{array}{r}12,851 \\
5,428 \\
5,866\end{array}$ & $\begin{array}{r}203003 \\
65672 \\
121426\end{array}$ & $\begin{array}{r}3003.10^{6} \\
997.10^{6} \\
1490.10^{6}\end{array}$ & $\begin{array}{r}54,529 \\
103,372 \\
67,710\end{array}$ \\
\hline \multicolumn{3}{|c|}{ Total zone } & 76 & 48596 & 8,027 & 390101 & $5490.10^{6}$ & 37,474 \\
\hline I & $\begin{array}{l}28 \\
29 \\
30 \\
31\end{array}$ & $\begin{array}{ll}\text { C } & \ldots \\
\text { D } & \ldots \\
\text { MD } & \ldots \\
\text { I } & \ldots\end{array}$ & $\begin{array}{r}30 \\
31 \\
37 \\
3\end{array}$ & $\begin{array}{r}4500 \\
47700 \\
24498 \\
400\end{array}$ & $\begin{array}{r}6,300 \\
1,741 \\
5,000 \\
26,666\end{array}$ & $\begin{array}{r}28350 \\
83045 \\
122488 \\
10664\end{array}$ & $\begin{array}{r}72.10^{6} \\
326.10^{6} \\
2337.10^{6} \\
33.10^{6}\end{array}$ & $\begin{array}{r}61,056 \\
44,352 \\
78,847 \\
171,316\end{array}$ \\
\hline \multicolumn{3}{|c|}{ Total zone } & 101 & 77098 & 3,172 & 244547 & $2768.10^{6}$ & 42,598 \\
\hline $\mathbf{J}$ & $\begin{array}{l}32 \\
33 \\
34\end{array}$ & $\begin{array}{l}\mathrm{C} \ldots \\
\mathrm{MD} \ldots \\
\mathrm{I} \ldots\end{array}$ & $\begin{array}{l}11 \\
45 \\
13\end{array}$ & $\begin{array}{r}1000 \\
38997 \\
6200\end{array}$ & $\begin{array}{l}4,909 \\
4,688 \\
5,307\end{array}$ & $\begin{array}{r}4908 \\
182.818 \\
32902\end{array}$ & $\begin{array}{r}4.10^{6} \\
1639.10^{6} \\
356.10^{6}\end{array}$ & $\begin{array}{r}89,649 \\
44,733 \\
123,867\end{array}$ \\
\hline \multicolumn{3}{|c|}{ Total zone . . . . . . . . } & 69 & 46197 & 4,776 & 220628 & $1999.10^{6}$ & 40,530 \\
\hline $\begin{array}{l}\text { Total } \\
\text { Total I } \\
\text { Total I } \\
\text { Total I }\end{array}$ & & & $\begin{array}{l}254 \\
409 \\
580 \\
183\end{array}$ & $\begin{array}{r}86132 \\
620087 \\
464681 \\
57895\end{array}$ & $\begin{array}{l}9,786 \\
7,113 \\
5,768 \\
6,200\end{array}$ & $\begin{array}{r}842916 \\
4410789 \\
3145167 \\
358947\end{array}$ & $\begin{array}{r}9449.10^{6} \\
198544.10^{6} \\
74403.10^{6} \\
5998.10^{6}\end{array}$ & $\begin{array}{l}22,370 \\
19,798 \\
16,997 \\
42,289\end{array}$ \\
\hline Total & & & 1426 & 1228795 & 7,127 & 8757919 & $288394.10^{6}$ & 12,018 \\
\hline
\end{tabular}

C : Peuplements clairs de basse altitude.

D : Peuplements denses.

MD : Peuplements moyennement denses.

I : Peuplements clairs d'altitude.
Altitude moyenne :

$950-1000 \mathrm{~m}$

$1000-1200 \mathrm{~m}$

$1200-1350 \mathrm{~m}$

$>1350 \mathrm{~m}$. 


\begin{tabular}{|c|c|c|c|c|c|c|c|c|}
\hline Zone & $\begin{array}{l}\text { Sous- } \\
\text { zone }\end{array}$ & $\begin{array}{l}\text { Peuple- } \\
\text { ments }\end{array}$ & $\begin{array}{c}\text { Nbre } \\
\text { placettes }\end{array}$ & $\begin{array}{l}\text { Nombre } \\
\text { de pins }\end{array}$ & $\begin{array}{c}\text { Nombre } \\
\text { de } \\
\text { nids/pins }\end{array}$ & $\begin{array}{l}\text { Popu- } \\
\text { lation } \\
\text { totale }\end{array}$ & Variance & $\begin{array}{c}\text { Erreur \% } \\
=\mathrm{t} \sigma / \mathrm{T}\end{array}$ \\
\hline \multirow{4}{*}{ A } & 1 & $\mathrm{C}$ & 22 & 6098 & 9,181 & 55990 & $171.10^{6}$ & 48,346 \\
\hline & 2 & $\mathrm{D}$ & 6 & 4700 & 4,000 & 18800 & $75.10^{6}$ & 112,860 \\
\hline & 3 & MD & 109 & 64287 & 4,091 & 263000 & $1477.10^{6}$ & 28,641 \\
\hline & 4 & I & 66 & 12588 & 3,257 & 41000 & $83.10^{6}$ & 44,441 \\
\hline Total & \multicolumn{2}{|l|}{ zone } & 203 & 87673 & 4,320 & 378790 & $1806.10^{6}$ & 21,990 \\
\hline \multirow{4}{*}{ B } & 5 & $\mathrm{C}$ & 32 & 4899 & 3,406 & 16687 & $14.10^{6}$ & 45,742 \\
\hline & 6 & D & 8 & 11200 & 1,125 & 12600 & $45.10^{6}$ & 122,984 \\
\hline & 7 & $\mathrm{MD}$ & 75 & 58095 & 1,653 & 96031 & $249.10^{6}$ & 32,864 \\
\hline & 8 & & 60 & 10698 & 3,500 & 37443 & $40.10^{6}$ & 33,782 \\
\hline Total & \multicolumn{2}{|l|}{ zone } & 175 & 84892 & 1,917 & 162761 & $348.10^{6}$ & 22,464 \\
\hline \multirow[b]{2}{*}{$\mathrm{C}$} & 9 & $\mathrm{C}$ & 29 & 5298 & 2,586 & 13701 & $9.10^{6}$ & 44,668 \\
\hline & 10 & MD & 13 & 8600 & 2,153 & 18515 & $19.10^{6}$ & 50,852 \\
\hline \multirow[t]{3}{*}{ Total } & \multicolumn{2}{|l|}{ zone. } & 42 & 13898 & 2,318 & 32216 & $28.10^{6}$ & 33,179 \\
\hline & 11 & & 59 & 15995 & 8,745 & 139875 & $667.10^{6}$ & 37,297 \\
\hline & 12 & & 140 & 196994 & 5,885 & 1159310 & $13940.10^{6}$ & 20,165 \\
\hline \multirow[t]{2}{*}{ D } & 13 & MD & 178 & 96378 & 8,252 & 795385 & $6271.10^{6}$ & 19,713 \\
\hline & 14 & $I \ldots$ & 72 & 11599 & 9,444 & 109543 & $357.10^{6}$ & 34,497 \\
\hline \multirow[t]{2}{*}{ Total } & & & 449 & 320966 & 6,867 & 2204113 & $21235.10^{6}$ & 12,958 \\
\hline & 15 & & 115 & 172994 & 5,147 & 890402 & $12426.10^{6}$ & 25,039 \\
\hline \multirow[t]{2}{*}{$\mathrm{E}$} & 16 & MD & 61 & 18196 & 16,967 & 308737 & 2. $347.10^{6}$ & 31,383 \\
\hline & 17 & & 29 & 3297 & 18,586 & 61283 & $177.10^{6}$ & 44,287 \\
\hline \multirow[t]{2}{*}{ Total } & zone. & 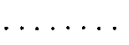 & 205 & 194487 & 6,481 & 1260422 & $14950.10^{6}$ & 19,207 \\
\hline & 18 & $\mathrm{C}$ & 81 & 23198 & 9,296 & 215652 & $4645.10^{6}$ & 63,208 \\
\hline \multirow[t]{2}{*}{ F } & 19 & & 14 & 17699 & 7,214 & 127679 & $1157.10^{6}$ & 57,278 \\
\hline & 20 & MD & 25 & 27600 & 8,040 & 221904 & $2775.10^{6}$ & 48,903 \\
\hline Total & \multicolumn{2}{|l|}{ zone } & 120 & 68497 & 8,252 & 565235 & $8577.10^{6}$ & 32,442 \\
\hline \multirow{4}{*}{$\mathrm{G}$} & 21 & & 24 & 11100 & 13,916 & 154468 & $736.10^{6}$ & 36,180 \\
\hline & 22 & & 72 & 152597 & 5,458 & 832873 & $3893.10^{6}$ & 14,983 \\
\hline & 23 & $\mathrm{MD}$ & 25 & 16700 & 10,760 & 179692 & $2022.10^{6}$ & 51,550 \\
\hline & 24 & & 27 & 6099 & 17,481 & 106622 & $529.10^{6}$ & 44,222 \\
\hline Total & \multicolumn{2}{|l|}{ zone } & 148 & 186496 & 6,829 & 1273655 & $7180.10^{6}$ & 13,173 \\
\hline \multirow{3}{*}{$\mathbf{H}$} & 25 & & 48 & 3693 & 11,083 & 40963 & $61.10^{6}$ & 38,514 \\
\hline & 26 & D & 15 & 23400 & 4,000 & 93600 & $1117.10^{6}$ & 76,055 \\
\hline & 27 & MD & 23 & $1449 \mathrm{c}$ & 8,347 & 121025 & $857.10^{6}$ & 50,071 \\
\hline \multirow[t]{2}{*}{ Total } & zone & & 86 & $415^{\circ}$ & 6,145 & 255588 & $2035.10^{6}$ & 35,300 \\
\hline & 28 & C & 22 & 4798 & 12,818 & $61 \overline{503}$ & $399.10^{6}$ & $6 \overline{7,230}$ \\
\hline \multirow{3}{*}{ I } & 29 & D & 41 & 36297 & 3,024 & 109763 & $358.10^{6}$ & 34,821 \\
\hline & 30 & MD & 35 & 15498 & 8,114 & 125751 & $868.10^{6}$ & 47,795 \\
\hline & 31 & I . . & 13 & 1000 & 25,000 & 24992 & $43.10^{6}$ & 56,674 \\
\hline Total & zone & & 111 & 57593 & 5,591 & 322009 & $1668.10^{6}$ & 25,366 \\
\hline & 32 & & 11 & 600 & 14,090 & 8447 & $5.10^{6}$ & 58,238 \\
\hline $\mathbf{J}$ & 33 & MD & 43 & 31596 & 9,372 & 296121 & $2308.10^{6}$ & 32,772 \\
\hline & 34 & I & 32 & 5600 & 11,156 & 62474 & $606.10^{6}$ & 80,383 \\
\hline Total & zone & & 86 & 37796 & 9,711 & 3670.42 & $2 \cdot 919.10^{6}$ & 29,439 \\
\hline$\overline{\text { Total }}$ & C & & 328 & 75679 & $9 \overline{9,3459}$ & 707286 & $6707.10^{6}$ & 22,926 \\
\hline Total & D & & 411 & 615881 & 5,2689 & 3245027 & $33011.10^{\circ}$ & 10,974 \\
\hline Total & MD & & 587 & 351449 & 6,9033 & 2. 426161 & $19193.10^{6}$ & 11,191 \\
\hline Total & I . . & & 299 & 50881 & 8,7136 & 443357 & $1835.10^{6}$ & 19,131 \\
\hline Total & vallée & & 1625 & 1093890 & 6,2363 & 6821831 & $60746.10^{\circ}$ & 7,081 \\
\hline
\end{tabular}




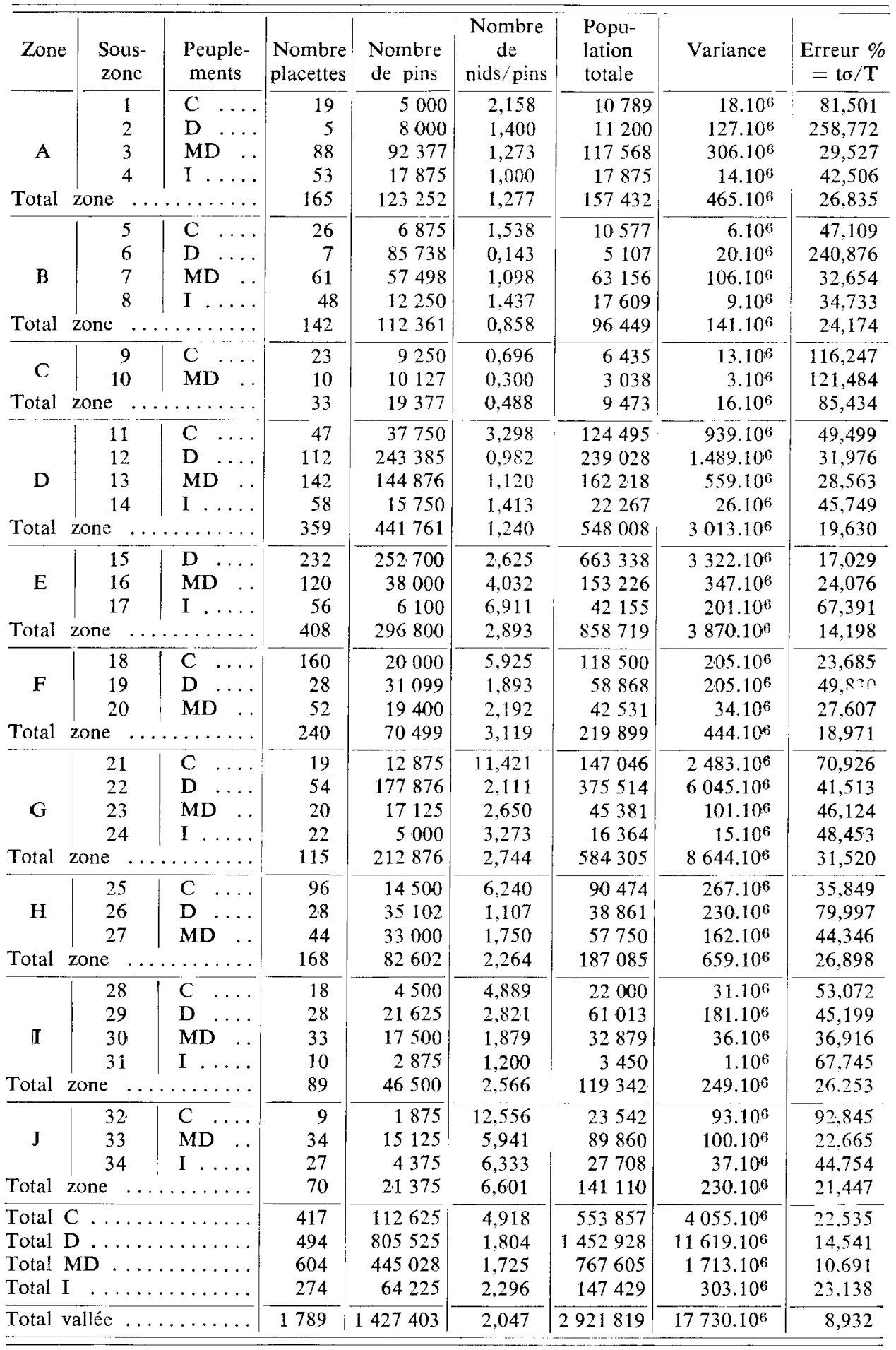


s'abaisse de 6822000 nids à 2922000 nids en 1970. La réduction est enregistrée de façon très nette dans toutes les zones et sous-zones (sauf la sous-zone 32 ); elle est également évidente sur la figure 7.

L'erreur statistique (8,9 p. 100) sur la population totale est en 1970 du même ordre que celle des années précédentes. Il en est de même pour les principaux types de peuplement (10 à 20 p. 100 en moyenne) et pour les différentes zones (20 à 30 p. 100). La précision est un peu plus faible dans les zones où l'intensité de sondage a été réduite; elle est améliorée dans les zones à échantillonnage hiérarchisée. Les limites d'erreur demeurent satisfaisantes pour l'estimation de la population de la vallée et acceptables pour suivre, zone par zone, les mouvements locaux des populations de processionnaires.

On observe en outre, sur la figure 7 , que la répartition des populations dans la vallée a tendance à se conserver d'année en année. En particulier, les populations sont plus abondantes sur le versant sud que sur le versant nord. Ce fait, également mis en évidence dans d'autres massifs forestiers (Mont Ventoux, Massif de Mora de Rubielos en Espagne) est particulièrement évident en 1970, où les zones les moins attaquées correspondent, par ailleurs, aux régions d’altitude où la fonte des neiges fut la plus tardive.

Le nombre de nids par pin est plus élevé dans les peuplements clairs que dans les peuplements denses. Toutefois ceux-ci représentent des effectifs de chenilles importants. Entre 1966 et 1968, la diminution de population affecte surtout les peuplements denses. Il est probable que ceux-ci, moins surpeuplés en 1965 que les zones de pins clairs et plus riches en feuillage, ont constitué des réservoirs permettant la survie de la population et assuré, en partie, la repopulation en 1967 des secteurs voisins.

Un certain nombre de résultats constatés sur l'évolution des populations d'un cycle à l'autre rappellent donc les phénomènes mis en évidence par l'étude parcellaire, notamment l'incidence des facteurs climatiques. Par ailleurs, ils font apparaître des liaisons entre les populations et le milieu physique et forestier. Celles-ci seront plus particulièment étudiées dans le paragraphe 2.2.3.

\subsection{Facteurs biotiques de mortalité}

\subsection{Parasites}

Les larves de deux espèces de Tachinaires ont été observées sur les chenilles récoltées : Phryxe caudata Rond. et Ctenophorocera pavida Meig.

Les données sur $P$. caudata ne permettent pas d'évaluer l'activité de cet insecte dans les différentes zones du massif forestier, car la plupart des larves quittent l'hôte à la mue L4-L5, avant les prélèvements de nids. Elles ont cependant permis à G. DuSAussoy d'étayer l'hypothèse que Phryxe présente, dans la vallée, deux types de développement (communication personnelle) :

- un premier, au-dessous de $1000 \mathrm{~m}$ et dans quelques points bien exposés d'altitude, caractérisé par la sortie de l'asticot à la mue L4-L5 de l'hôte ;

- un second, dans les zones plus élevées et dans les expositions moins favorables, caractérisé par un arrêt de développement au premier stade avec reprise 

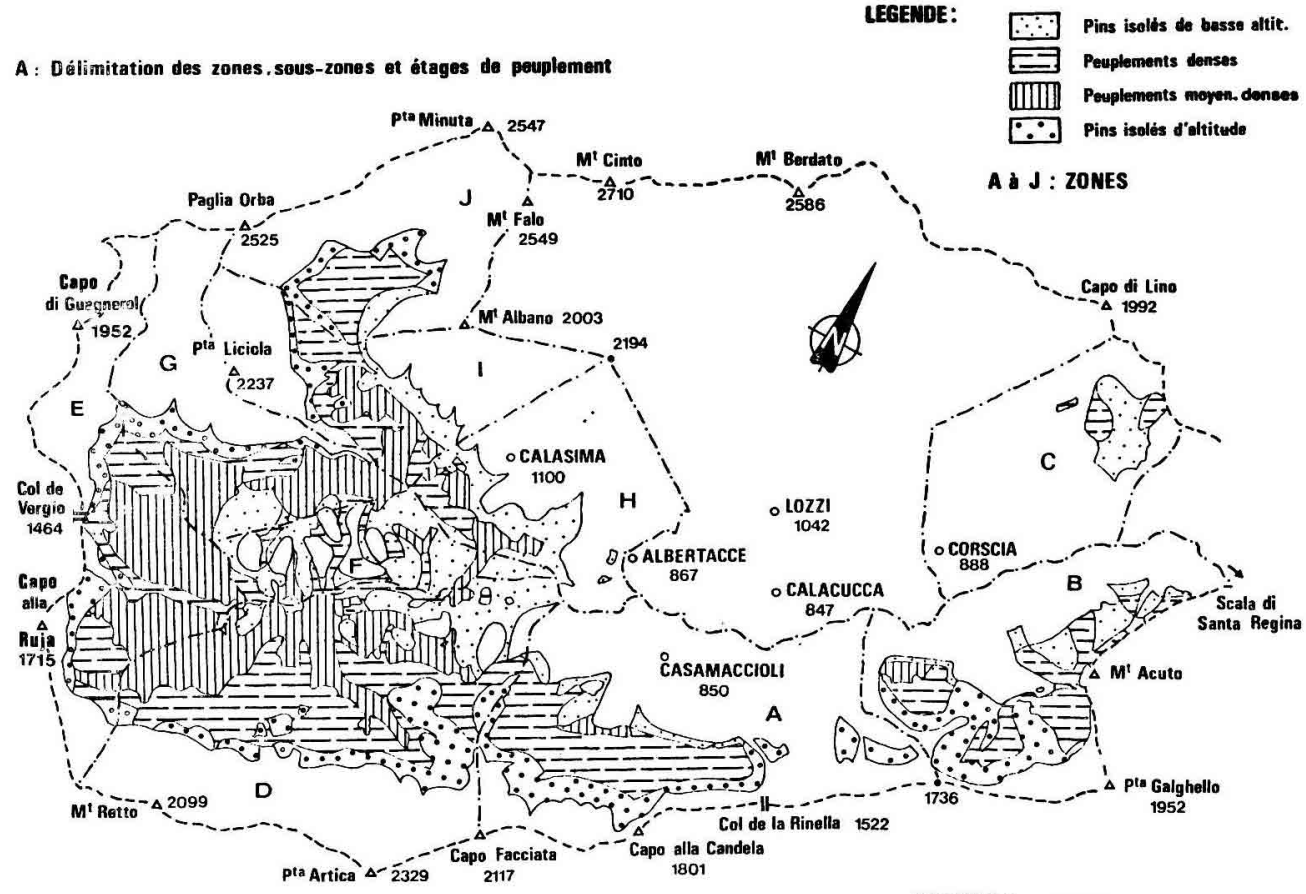

LEGENDE :

B : Nombre de nids recensés pour chaque cycle

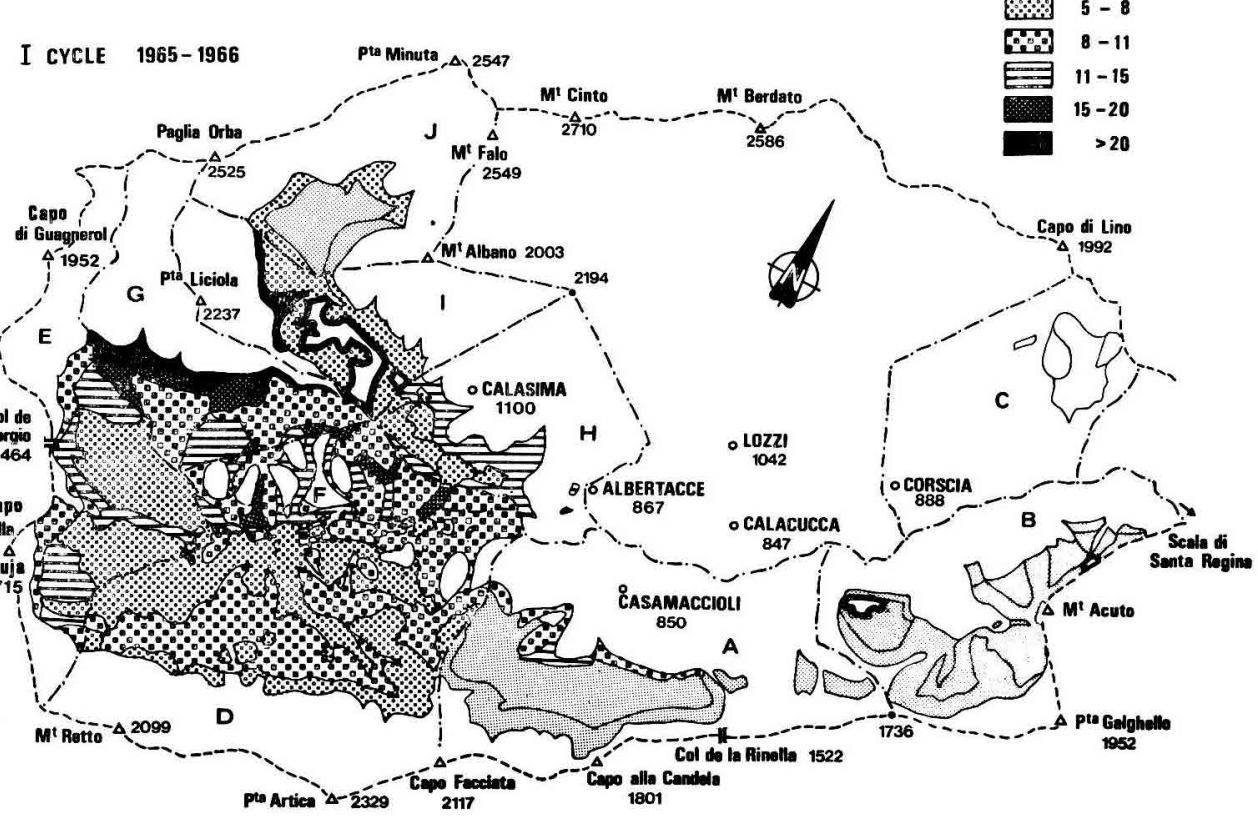

FIG. 7

Représentation cartographique du nombre moyen de nids par pin recensés sur l'ensemble de la vallée,

dans les différentes sous-zones pour les trois cycles étudiés.

Maping of mean nest number on a pine throughout the valley area, in different sub-area for the three studied life cycles. 
B: Nombre de nids recensés pour chaque cycle

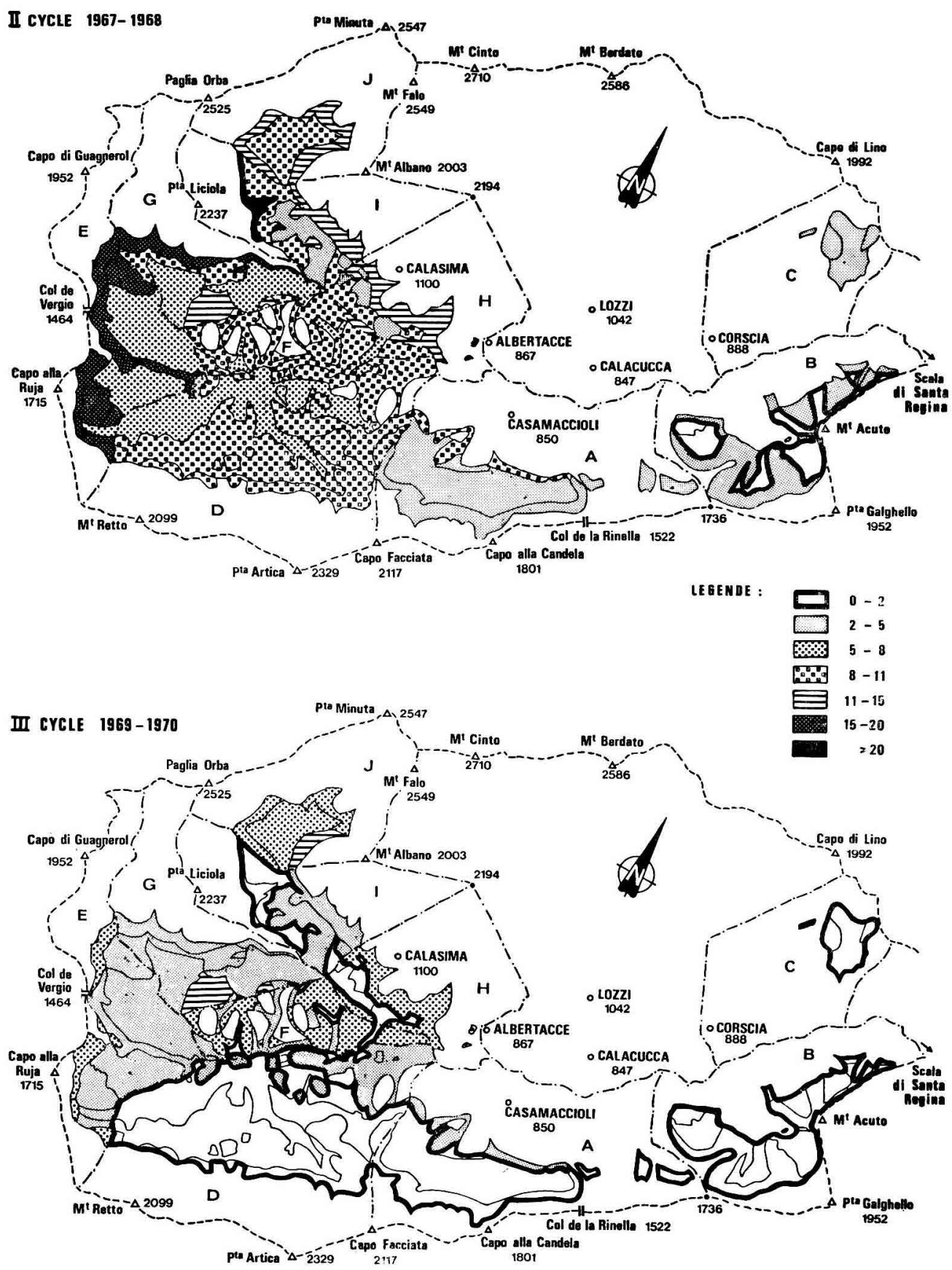


d'activité des larves au $5^{\circ}$ stade de l'hôte. Dans ce dernier cas, la sortie des asticots s'effectuerait dans le sol, peu après l'enfouissement des chenilles.

C. pavida, polyphage, parasite également le cul-brun (Euproctis phaeorrhea Don.), lequel est présent dans le Niolo, localisé, en période de latence, dans les peuplements d'Epine-Vinette de l'Etna, Berberis aetnensis C. Presl. Ces peuplements sont situés pour la plupart à $1500-1600 \mathrm{~m}$ au-dessus de la forêt, près des bergeries. Le cul-brun envahit et dévaste, périodiquement, la châtaigneraie du Niolo $(800-900 \mathrm{~m})$. Le parasitisme observé sur la processionnaire peut donc être lié à la dynamique du Cul-brun. Effectivement, C. pavida, déjà observée sur la processionnaire du pin en 1966 et 1968 dans certaines zones situées au voisinage des peuplements d'Epine-Vinette attaqués par E. phaeorrhea, a été trouvée en 1970 pour la première fois dans nos parcelles d'étude, et dans 3 autres localisations situées au cour de la pineraie, alors que parallèlement la présence du cul-brun paraissait se généraliser dans la châtaigneraie. Mais il est plus probable que l'importance de cette tachinaire en 1970 est due surtout au retard phénologique des processionnaires au cours du cycle de 1969-1970; en effet, la coïncidence entre la processionnaire et le parasite est rarement assurée; E. BiLıotTI (1959) constate que $C$. pavida ne peut parasiter les processionnaires que dans los localisations exceptionnelles comme Valdo-Niello où le développement de l'insecte est tardif. Et, effectivement, les chenilles parasitées observées en 1966 et 1968 provenaient toutes de peuplements d'altitude du versant nord où l'évolution de la processionnaire est très lente.

\subsection{Maladies}

Il s'agit essentiellement de la virose cytoplasmique à polyèdres de la processionnaire du pin (Reovirus).

Le cycle 1965-1966, correspondant à la culmination de la gradation, fut le seul où cette maladie s'est manifestée de façon épidémique. Elle fut retrouvée à un niveau plus faible en 1967-1968 et seulement à l'état endémique en 1969-1970. La figure 8 résume les observations de 1966 . Elle montre :

- qu'il y avait moins de cas de virose dans les expositions nord que dans les expositions sud;

- que, ceux-ci diminuaient en fonction de l'altitude;

- qu'ils augmentaient avec le niveau d'infestation.

Une corrélation significative $(r=0,882)$ fut enregistrée entre le niveau de population moyen (nombre de nids par pin) des zones en 1966 et le pourcentage de nids présentant des cas de virose. Aucune relation ne fut, par contre, établie entre ce pourcentage et la réduction de population observée entre 1966 et 1968 .

Ces résultats confirmant ceux de l'étude parcellaire suggèrent que, dans une zone d'altitude telle que la vallée du Niolo, la virose ne peut se déclarer que dans des circonstances bien déterminées du cycle de gradation et que, même dans ce cas, elle pourrait affecter essentiellement les zones les plus basses et les plus ensoleillées. Ils montrent que la virose est probablement à considérer plus comme une conséquence, dans certaines conditions de milieu favorable de la surpopulation, que comme un facteur par lui-même important. 


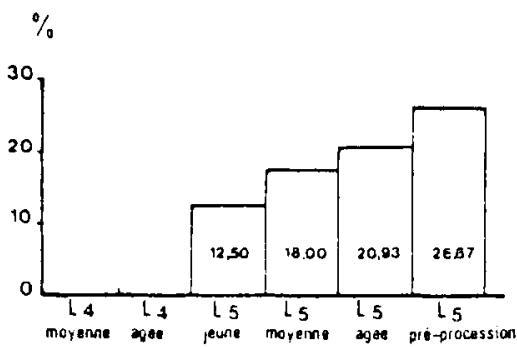

2 Orientation

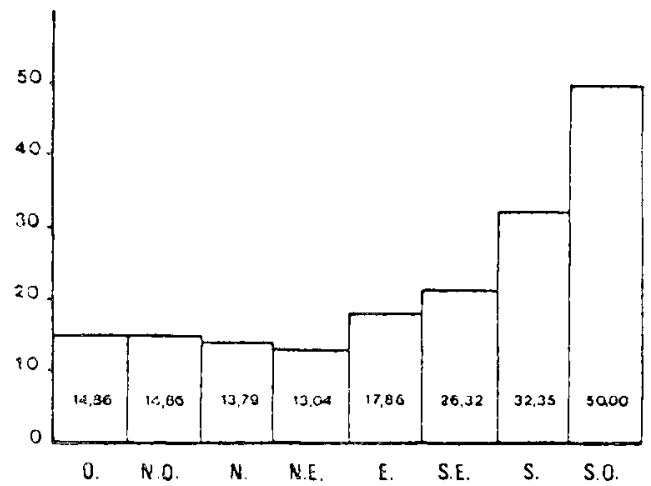

3 Aititude

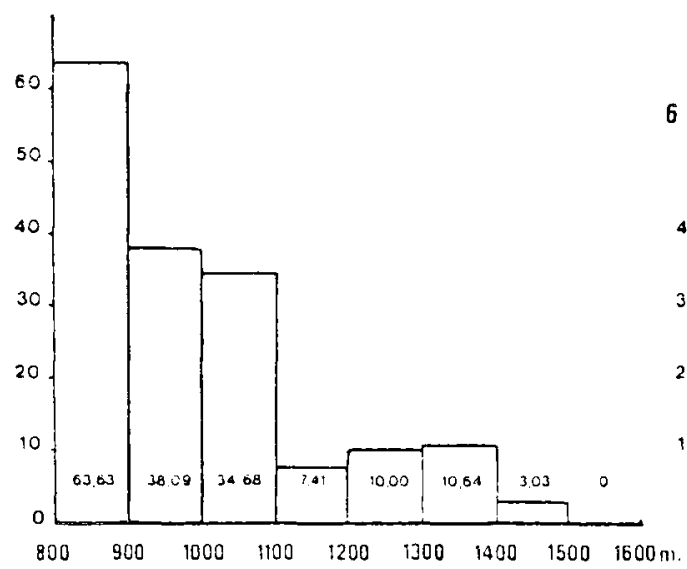

FIG. 8

Pourcentage de nids présentant des cas de viroses déclarées en fonction de divers facteurs écologiques en 1966.

Percentage of nest with virus disease following some ecological factors in 1966.
5 Zones

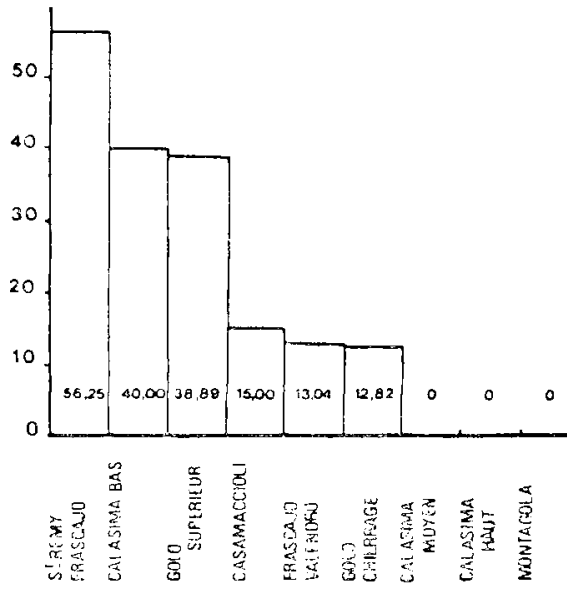

6 Indiga dinígention

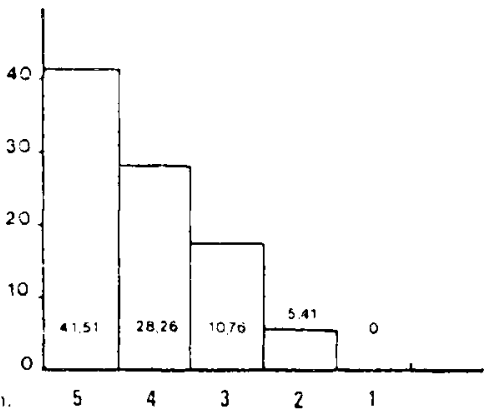




\subsection{Interactions entre les niveaux de populations, leurs fluctuations et les caractéristiques de l'environnement forestier}

Les résultats présentés ici ne concernent que les corrélations simples entre les niveaux de population (nombre de nids par pin) et les données recueillies sur l'environnement forestier.

Deux études ont été effectuées :

- l'examen des corrélations pour chaque cycle, sur l'ensemble des pins observés en tenant compte du découpage en sous-zones (correlation intra et inter) ;

- celui des corrélations pour l'ensemble des années entre les moyennes par souszone des variables considérées. Compte tenu du mode d'échantillonnage (les pins observés dans chaque sous-zone sont tirés au hasard à chaque cycle), les sous-zones constituent en effet les plus petites unités permettant d'apprécier les fluctuations des niveaux de populations d'année en année.

\subsection{Corrélations mises en évidence au niveau des pins pour chaque cycle}

Les conditions du milieu sont souvent trop homogènes à l'intérieur d'une même sous-zone, ou le nombre de données insuffisant, pour que les liaisons observées soient toujours significatives. Les principaux faits à retenir sont les suivants :

- il existe une corrélation négative entre le nombre de nid par pins et le cosinus de l'exposition traduisant les expositions nord, positive avec le sinus indiquant les expositions est. L'exposition sud-est paraît donc bien être la plus favorable au développement des attaques de processionnaires : au contraire, les régions en exposition nord-ouest sont les moins peuplées;

- peu de résultats significatifs sont obtenus avec l'altitude ; les corrélations sont tantôt négatives, tantôt positives traduisant vraisemblablement l'effet antagoniste de plusieurs facteurs (mortalité, vols d'adultes, diapause) ;

- plus un peuplement est dense, plus il est composé d'arbres élevés et comporte d'étages de pins, moins il est attaqué par les processionnaires;

- de la même façon, la corrélation entre le nombre de nids par pin et le nombre de pins de la placette est généralement négative. Ces constatations confirment les observations biologiques selon lesquelles un peuplement dense de futaie serait moins attaqué qu'un peuplement clair de clairière avec des pins se découpant en silhouette (DEMoLIN, 1962, 1969) ;

- plus un pin est haut, et d'un diamètre important, plus il abrite de nids. Ce résultat n'est qu'apparemment en contradiction avec la constatation faite au niveau du peuplement. Il s'explique par le comportement des adultes (DEMolin, 1969) : une parcelle de grands arbres fait généralement partie d'une futaie peu attractive mais plus l'arbre échantillonné est grand, plus il se distingue des voisins et est capable d'attirer les femelles.

\subsection{Corrélations mises en évidence sur les moyennes des sous-zones}

La comparaison des valeurs moyennes par sous-zones, sur les trois cycles, met en évidence une corrélation positive significative entre la population de l'année 1966 et celle de l'année 1968 ; entre la population de l'année 1968 et celle de l'année 1970. 
Donc, à chaque cycle, la population d'une sous-zone dépend de celle du cycle précédent. Dans l'ensemble, les régions les plus peuplées ont tendance à le demeurer et le maintien de la répartition des populations dans la vallée précédemment observée est confirmée. En particulier, les zones en exposition sud, restent. d'un cycle à l'autre, significativement plus peuplées que les expositions nord.

L'analyse de la variation des populations entre 1966 et 1968 (nombre de nids 1968 - nombre de nids 1966, nombre de nids 1968 /nombre de nids 1966) en fonction du nombre de nids 1966 montre en outre que plus les sous-zones étaient peuplées en 1966, plus la diminution de population fut importante. Par contre, aucune corrélation significative n'est enregistrée entre la diminution de population de 1968 à 1970 et le niveau de population atteint en 1970 .

En fonction des facteurs du milieu tels que l'exposition et l'altitude, l'analyse des modifications de population d'un cycle à l'autre ne montre pas de relations simples : la complexité des interactions entre ces facteurs et l'existence de phénomènes sousjacents, mortalité et déplacements des insectes, de nature différente, peuvent en être la cause. Une étude plus détaillée de ces interactions, en collaboration avec C. Milcier, par différentes techniques d'analyses multivariates, est en cours.

\subsection{Discussion}

L'étude transcyclique met en évidence l'abaissement progressif au cours des cycles 1967-1968 et 1969-1970 du niveau de population, après la culmination de gradation en 1965-1966. Ces fluctuations de population correspondent bien à celles mises en évidence par l'étude parcellaire et confirment la généralité d'un certain nombre de phénomènes observés lors de celle-ci.

- Influence de la densité des populations : les zones les plus peuplées au cours de l'année 1966 furent celles où la réduction de population fut la plus importante au cours des années suivantes.

- Progression de l'infestation dans les zones d'altitude en 1967.

- Présence généralisée d'une virose sur les fortes populations de 1965-1966, limitée, quant à sa manifestation épidémique, aux zones les plus infestées et les mieux exposées de la vallée.

- Impact moins important de cette maladie durant le second cycle et manifestation seulement à l'état endémique en 1970.

- Confirmation de certains points de la biologie de Phrye caudata et de Ctenophorocera pavida.

- Populations plus faibles en exposition nord quen exposition sud. Importance de ce facteur en 1970.

- Influence moins évidentc et plus irrégulière de l'altitude, vraisemblable toutefois en 1970 .

Mais ces résultats permettent en outre de constater des faits importants :

- les populations des différentes zones et sous-zones sont liées d'année en année. La répartition de l'insecte dans la vallée a tendance à se conserver d'une génération 
à l'autre comme si la densité des populations de processionnaires était, au même titre que les différentes variables dendrométriques ou sylvicoles, une caractéristique même des divers peuplements du massif;

- les niveaux de population de l'insecte sont étroitement liés aux conditions physiques et aux caractéristiques du milieu forestier.

\section{Conclusion}

L'approche utilisée dans la Vallée du Niolo s'inspire des techniques empruntées à la démographie humaine (tables de mortalité) développées notamment par l'école canadienne de R.F. Morris et des techniques d'enquêtes forestières, utilisées par AUER pour l'étude des populations de tordeuse du Mélèze sur des vallées entières (A. KAELIN \& C. Auer, 1954 ; C. Auer, 1968).

Les résultats montrent comment l'utilisation simultanée des deux niveaux d'investigation permet d'obtenir, non des preuves absolues mais des hypothèses largement étayées sur la façon dont se produit l'évolution d'un déprédateur.

Ils mettent cependant en lumière l'insuffisance de ces deux méthodes utilisées isolément, pour l'étude de la dynamique des populations d'un insecte :

- les techniques de "tables de mortalité » sont mal adaptées à la processionnaire du pin, dans la mesure où cet insecte n'effectue pas la totalité de son développement sur l'arbre, et où la succession des générations est perturbée par la diapause. D'autre part, sa répartition sur le végétal nécessite des méthodes d'échantillonnage laborieuses. Elles ne fournissent, à cause des surfaces d'étude restreintes qu'une image statique et non dynamique de l'évolution des populations, qui ne prend pas en compte les déplacements de l'insecte à l'intérieur d'un massif. En outre, la traduction en termes de mortalité de l'action de tous les facteurs affectant les différents stades et de leurs interactions paraît illusoire, et risque d'être entachée d'erreurs considérables, tant sur le plan statistique que du point de vue de la représentativité des phénomènes biologiques.

Des évaluations systématiques des niveaux de population et de l'incidence des facteurs de mortalité à chaque stade, sont cependant indispensables pour préciser la nature des principaux agents de régulation et pour mettre en évidence les stades "critiques" de la vie de l'insecte. Dans le cas présent, l'étude intracyclique a, seule, permis de mettre en évidence :

- le rôle prépondérant de la surpopulation au cours du cycle 1965-1966;

- l'importance de la mortalité hivernale au cours du cycle 1969-1970;

- l'intervention simultanée des deux facteurs au cours du cycle 1967-1968; cycle ;

- Ia faible incidence des premiers stades larvaires sur la mortalité totale du

- le rôle secondaire des parasites des œufs et à un moindre degré des parasites des larves durant les six années d'observation.

La technique d'enquête forestière, si elle ne permet pas une étude aussi détaillée des processus de mortalité fournit une information de portée plus générale. Elle 
permet de comprendre les grands mouvements de population qui se produisent d'année en année sur des territoires importants. Elle est particulièrement adaptée à leur intreprétation en fonction des situations climatiques et des conditions de peuplements. Dans le cas présent, elle a permis de mettre en évidence des relations étroites entre l'importance des attaques et les caractéristiques des peuplements et du site qui montre le rôle déterminant de la structure et de la nature des peuplements pour le maintien et la répartition de l'espèce, ouvrant ainsi des perspectives pour la limitation des populations de ravageur par la conduite sylvicole. En outre, l'utilisation des données ainsi acquises, si elles sont étayées par des études plus détaillées et par des travaux biologiques approfondis sur les facteurs de régulation, peut conduire à la construction de modèles de l'évolution de l'insecte permettant d'établir à n'importe quel moment de la gradation un diagnostic de l'état des populations, de faire un pronostic concernant leur évolution et de simuler l'utilisation des différentes méthodes d'intervention y compris des techniques préventives d'aménagement forestier.

Ces trois premiers cycles biologiques étudiés soulignent limportance d’un certain nombre de facteurs pour la régulation des populations de processionnaire du pin dans les vallées d’altitude de la Corse. L’importance numérique des populations, le rapport entre la densité de celles-ci et le végétal-hôte ainsi que les facteurs climatiques, tant par leur action directe que par leur incidence sur la phénologie de la processionnaire, apparaissent comme des facteurs essentiels. Ces facteurs navaient pas toujours reçu, dans les études sur la processionnaire du pin, lattention qu'ils méritent. Par contre, les agents biotiques sur lesquels avaient porté jusqu'à présent l'essentiel des études biologiques ne paraissent avoir qu'une action limitée sur le devenir des populations de processionnaire dans la vallée du Niolo. Les taux de parasitisme sont relativement constants malgré les fluctuations des populations de l'hôte. L'action de la virose paraît se manifester essentiellement au cours des périodes de surpopulation.

Sur le plan de la lutte biologique, ces résultats sont, au moins à première vue, peu encourageants. Il existe à l'état naturel, dans la vallée dı Niolo, un effectif de plusieurs milliards de parasites très supérieurs à celui qui peut être fourni par des élevages d'entomophages, et leur incidence sur le devenir des populations n’en demeure pas moins restreinte. Leur utilisation sur une telle population de processionnaires se développant depuis des centaines d’années en équilibre avec son milieu forestier ne peut se concevoir qu'en suivant une stratégie écologique rigoureusement calculée en fonction de la gradologie de l'insecte et de sa faune associée dans le massif forestier.

Par contre l'étude montre pour la première fois comment l'intensité des attaques et l'évolution des populations sont liées à la structure même et à la composition spécifique de chaque massif forestier; elle constitue une première approche pour la constitution et l'aménagement de boisements qui par eux-mêmes se défendent mieux contre les attaques d'insectes. 


\section{Summary \\ Pine processionary dynamics in the Niolo Valley, Corsica, during the cycles 65-66, 67-68, 69-70. \\ The role of several forest environment characteristics}

This article is an update of the demographic data collected during 3 study cycles $(65-66,67-68,69-70)$ about the pine processionary in the Niolo Valley, Corsica. Simultaneous use of two investigation levels with the plot scale data on the one hand, with the forest scale data on the other hand allows to make strong assumptions, if not absolute certainties, about the evolution of this insect over those 6 years.

The results show the statistically significant (5 p. 100 confidence intervals) variations of the population size and suggest that:

- the population size which peaked in 1966 (8 800000 nests) gradually decreased in 1968 (6800000 nests) and 1970 (2900 000 nests);

- the most populated areas in 1966 incurred the largest decrease in population size during the following years;

- the large population in 1965-1966 went along with a virus disease. The disease was only restricted in its epidemic nature to low altitude or well exposed areas and was only found in an endemic stade later on;

- North West facing areas are the least populated and South East ones are the most favorable to the processionary;

- for the 6 years studied, mortality during the first instars wasn't ever likely the modify noticeably the evolution of the population;

- in the same way, the parasites of the processionary during its aerian phase (mostly egg parasites) do not seem to have significantly influenced the recorded mortality;

- in 1965-1966, deaths mostly occurred before winter, in several plots they were due to a virus. The larger mortality rates were in the most overpopulated plots;

- in 1969-1970, deaths were due to winter and were unrelated to the population size. The populations size decrease was larger in North West facing and high altitude plots;

- the 1968-1969 cycle shows an intermediary scheme. The caterpillars were affected by a tough winter but wa also recorded some prewinter deaths, in some plots, due to overpopulation, as in 1965 ;

- the analysis also shows the links between the population size and the major forest characteristics. This relations have to be studied more thoroughly to make forests more resistante to Pine Processionnary.

\section{Références bibliographiques}

AUER C., 1968. Erste Ergebnisse einfacher stochastischer Modelluntersuchungen über die Ursachen der Populationsbewegung des grauen Lärchenwicklers Zeiraphera diniana Gn. (=Z. griseana HB.) im Oberengadin, 1949/1966. Z. angew. Entomol., 62 (2), 202-235.

BiLıorti E., 1958. Les parasites et prédateurs de Thaumetopoea pityocampa Schiff. (Lepidoptera). Entomophaga, 3 (1), 23-24.

CAMpbell R.W., 1967. The analysis of numerical change in Gipsy moth population. For. Sci. Monogr., 15-33.

Demolin G., 1962. Comportement des adultes de Thaumetopoea pityocampa Schiff. C.R. Acad. Sci., 255, 2838-2839.

Demolin G., 1969. Comportement des adultes de Thaumetopoea pityocampa Schiff. Dispersion spatiale, importance écologique. Ann. Sci. for., 26 (1), 81-102.

Demolin G., 1969. Bioecologica de la Processionaria del pino, Thaumetopoea pityocampa Schiff. Incidencia de les factores climaticos. Bol. Serv. Plagas for., 12 (23), 9-24. 
Dusaussoy G., Geri C., 1969. Etude des fluctuations du niveau de population de la processionnaire du pin dans la vallée du Niolo en Corse. Dispositif d'étude et premiers résultats. Ann. Sci. for., 26 (1), 103-125.

Geri C., 1971. Etude du niveau de population de Thaumetopoea pityocampa Schiff. dans la vallée du Niolo en Corse. Notes préliminaires sur les cycles biologiques (19651966 et 1967-1968). Ann. Zool. Ecol. anim., 2 (2), 67-87.

Geri C., 1980. Applications des méthodes d'études démécologiques aux insectes défoliateurs forestiers. Cas de Diprion pini L. (Hyménoptère Diprionidae). Dynamique des populations de la processionnaire du pin Thaumetopoea pityocampa Schiff. (Lépidoptère Thaumetopoeïdae) dans l'île de Corse. Thèse doct. Etat, Orsay, Université Paris-Sud, $260 \mathrm{p}$.

Geri C., 1980. Etudes sur les populations de processionnaire du pin (Thaumetopoea pityocampa Schiff., Lepidoptera Thaumetopoeidae). In : Grison P. (ed.), Etudes entomologiques et ethnologiques dans le Niolo. Ecol. méditerr., 6, 151-172.

Hansen M.H., Hurwitz W.N., Madow W.G., 1953. Sample survey methods and theory. In : Methods and applications, 1. New York, K. Wiley \& Sons, 638 p.

KaElin A., Auer C., 1954. Statistische Methoden zur Untersuchungen von Insektenpopulationen. Z. angew. Entomol., 36, 241-283, 423-461.

MORRIS R.F., 1955. The development of sampling techniques for forest insect defoliators with particular reference to the Spruce budworm. Can. Zool., 33, 226-294.

MORRIS R.F., 1957. The interpretation of mortality data in studies on population dynamics. Can. Entomol., 89 (2), 49-69.

Morris R.F., 1959. Single factor analysis in population dynamics. Ecology, 40, 580-588.

Morris R.F., 1963. Predictive population equations based on key factors. Mem. Entomol. Soc. Can., 32, 16-21.

Morris R.F. (ed), 1963. The dynamics of epidemic spruce budworm populations. Mem. Entomol. Soc. Can., 31, 332 p.

Morris R.F., Miller C.A., 1954. The development of life tables for the Spruce budworm. Can. J. Zool., 32, 283-301.

SNEDECOR G.W., 1956. Statistical methods applied to experiments in agriculture and biology. Ames Iowa, Iowa State University Press, 534 p.

Varley G.C., Gradwell G.R., 1960. Key factors in population studies. J. anim. Ecol., 29, 251-273.

WELLINGTON W.G., 1964. Qualitative changes in population in unstable environments. Can. Entomol., 96 (1/2), 436-451.

Wellington W.G., 1965. Some maternal influences on progeny quality in the western tent caterpillar, Malacosoma pluviale Dyar. Can Entomol., 97 (1), 1-14. 